\title{
Running-In of Systems Protected by Additive-Rich Oils
}

\author{
Rob Bosman • Dirk Jan Schipper
}

Received: 19 July 2010/ Accepted: 6 October 2010/Published online: 21 October 2010

(c) The Author(s) 2010. This article is published with open access at Springerlink.com

\begin{abstract}
Recent research on mild wearing systems running under boundary lubrication conditions focus more and more on the role of the nano-crystalline layer present at the surface of the components in contact. This layer has a typical thickness of a few tenths of nano-meters up to a few microns depending on the operational conditions. The role of this layer with respect to wear is, however, still unclear as well as its mechanical behavior. In this study, a first step is made in incorporating this type of layer into a wear model. Using an elasto-plastic semi-analytical-method the effect of different material behaviors reported through out current literature for the nano-crystalline layer on wear is studied. From the results it can be concluded that the effect of this mechanically altered layer has an important influence on the wear of the system, especially during the initial phase of running.
\end{abstract}

Keywords Boundary lubrication wear - Stress analysis . Oxidative wear

\section{Introduction}

The wear of components is of great interest to industry and society and therefore a lot of research is conducted on this topic. The publications range from developed (pragmatic) wear equations to written descriptions about the wear of certain systems, for a review the reader is referred to the article of Meng and Ludema [1]. As can be concluded from this article, many different equations are used and still one of the most commonly used is the Archard/Holm equation

R. Bosman $(\bowtie) \cdot$ D. J. Schipper

University of Twente, Enschede, The Netherlands

e-mail: r.bosman@utwente.nl
[2], where it is assumed that the wear is linear related to the sliding distance and normal load. Currently this model is most often used, or a derivative of it, enabling it to be incorporated within a numerical method such as BIM and FEM, see, e.g., [3-5]. This type of models yields good results if the appropriate specific wear coefficient is used and appropriate care is taken in chosen the right incremental steps for the sliding distance and load. However, it does not give insight into the wear mechanism behind material removal. The driving force behind the wear of materials is still a topic which is researched intensively in literature. The research is currently focusing more toward the micro-/nanolevel, as it is becoming clearer that the friction and wear in systems are determined in the first few nano-/micrometers underneath the surface. Looking at systems operating in boundary lubrication conditions extensive grain refinement down to tenths of nanometer is seen near the surface and different plastic material properties can be expected through the Hall-Petch relationship and the inverse Hall-Petch relationship [6, 7], e.g. material hardening or softening, respectively. Both of these theories are based upon physical hypotheses. The alleged mechanism behind the Hall-Petch relationship is the pile up of dislocations/grain, refinement, e.g., dislocation density increase. Since in coarse grain material the main plasticity mechanism is dislocation diffusion, the energy needed for this increases and thus the material is hardened. However, if the grain size is reduced to a level at which every crystal can only contain a very limited number of dislocations no pile up occurs and thus hardening due to grain refinement/dislocation pile up is limited. If now the grain size is reduced further a different types of plasticity mechanism is suggested; grain boundary slip rather than dislocation diffusion [8], e.g., the grains themselves are not deforming but the lattice as a total and grains start slipping over each other. This mechanism would 
promote super plasticity at relatively low temperatures, suggesting that a softer and more ductile layer is formed at the surface preventing the brittle and hard (sub-surface) bulk material from yielding. Other authors, however, claim an increase of hardness toward the surface [9], suggesting that the Hall-Petch relationship still holds even at submicron grain sizes. Both of these theories are consistent with theoretical and experimental studies performed at $\mathrm{n}$ (ano)-c(rystalline) materials [10-17]. The influence of this gradual increase and/or decrease of the yield stress on wear are currently not treated in a theoretical manner. In this article a first step to include the NC-layer, which are observed in experiments performed, is taken. The formation of this layer will not be dealt with, and it is assumed that the layer is stable after running-in conditions [18] having a constant thickness and grain size.

The wear model used in this study was first discussed by Nelias et al. [19] based on a semi-analytical elasto-plastic contact code. The SAM code was first developed by Jacq et al. [20] and later on adapted to include friction by Nelias et al. [21]. In the latter study, it is stated that there is a threshold equivalent plastic strain at which the material will start tearing. The formed crack will then grow toward the surface enclosing a volume which will form a wear particle. This method is used successfully to study two academic situations for a smooth surface with a high load and a lowly loaded isotropic rough surface [19]. In both the situations an overall coefficient of friction of 0.4 is assumed. It was shown that during the first contact cycles the surface can either run in (smoothening of the rough surface) or run out (roughening of the smooth surface). In the current model a similar approach is used; however, a local coefficient of friction based on the effectiveness of the lubricant to protect the surface is used.

It is suggested that a lubricated system running under boundary lubrication conditions can be modeled according to Fig. 1; with a system consisting of three layers [22]. The top layer is a physically/chemically adsorbed layer forming the first line of defense against high friction and extensive wear and is meant to provide protection under mild conditions. This layer is adsorbed on a harder thin chemically reaction layer, which is, however, still softer than the bulk material or the NC-layer. The chemical layer is typically built up from oxides formed on the surface and chemical products originating from the additives present in the oil, which have reacted with the oxide on the surface forming a complex structure which typically consists of an amorphous glass-type of material. Underneath this layer a metallurgical altered NC-layer exists, which is formed through high strain rates under high hydrostatic pressures. This layer is the final line of defense protecting the less ductile bulk material against tearing. The current model will focus mainly on the role of the chemical reacted layer

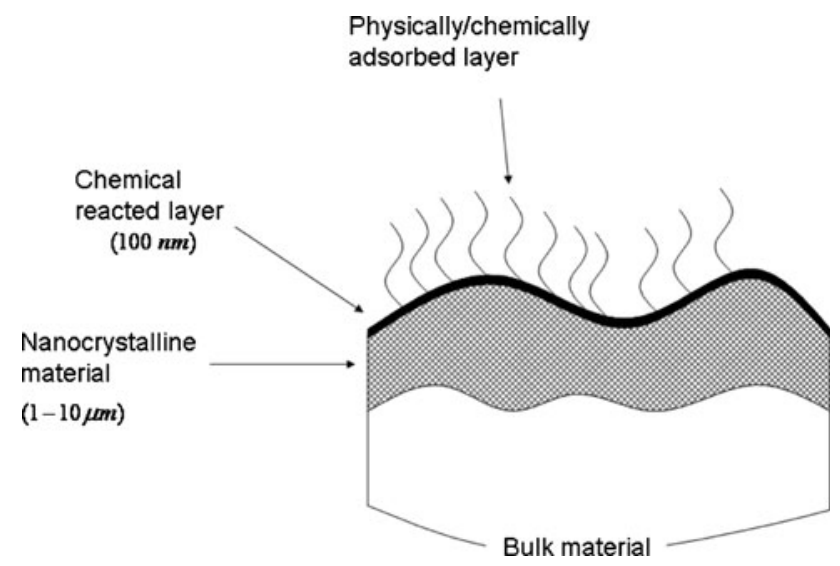

Fig. 1 Layers present at the surface of a run in system. The top layer is a physically/chemically adsorbed layer which only withstands very mild operational conditions. The second layer is a chemical layer which is a mixture of oxides and chemical products of the lubricant. The third layer is a nano-crystalline layer formed at the top of the bulk material by severe plastic deformation under large hydrostatic pressure

and NC-layer. The wear model will be based upon a SAM model and will be discussed briefly followed by a short discussion on the material model used for the NC-layer and finally the concept of the complete wear model will be discussed after which two examples will be shown, demonstrating the practical application of the current model.

\section{Contact Model}

The system built up in Fig. 1 may suggest that a layered contact model is needed. However, through some welleducated simplifications this is not necessary. First the chemical reacted layer, which is very thin in comparison with the contact patch size used in this study, will be neglected in the normal direction (e.g., the direction in which the normal load is applied). The chemical layer in systems operating under conditions studied is approximately 100-nm thick and has a Young's modulus of $80 \mathrm{GPa}$, as shown in Table 1. The data used for the

Table 1 Input parameters layered calculation

\begin{tabular}{ll}
\hline Input parameter & Value \\
\hline$E_{\text {coating }}$ & $80 \mathrm{GPa}$ \\
$E_{\text {substrate }}$ & $210 \mathrm{GPa}$ \\
$v_{\text {coating }}$ & 0.3 \\
$v_{\text {substate }}$ & 0.3 \\
$F_{\mathrm{N}}$ & $0.6 \mathrm{~N}$ \\
$\mu$ & 0.1 \\
$h_{\text {coating }}$ & $100 \mathrm{~nm}$ \\
\hline
\end{tabular}


micro-geometry are interference microscope measurements with a lateral resolution of $1 \mu \mathrm{m}$. To estimate the exact effect of the layer on the stress state and contact pressure the interference microscopy measurement shown in Fig. 2 is used combined with the theory presented in [23]. In

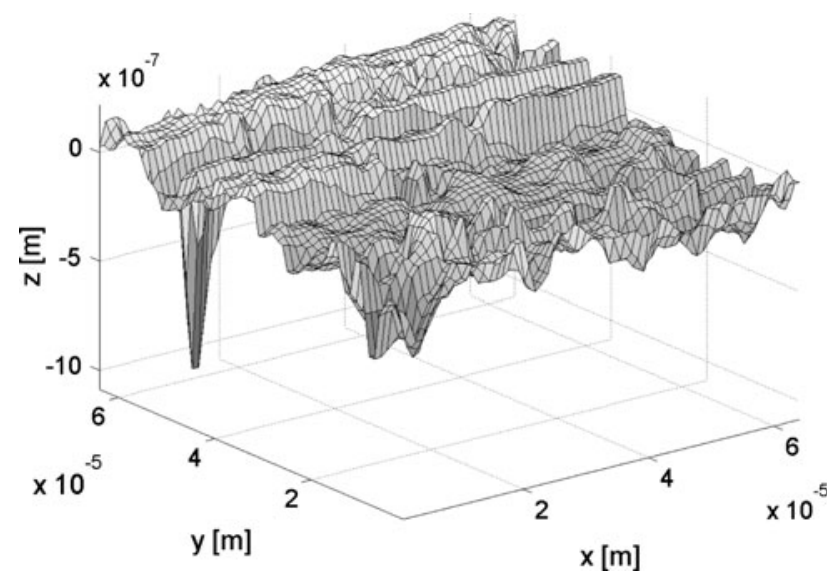

Fig. 2 Interference microscopy measurement used in the layered calculations

(a)
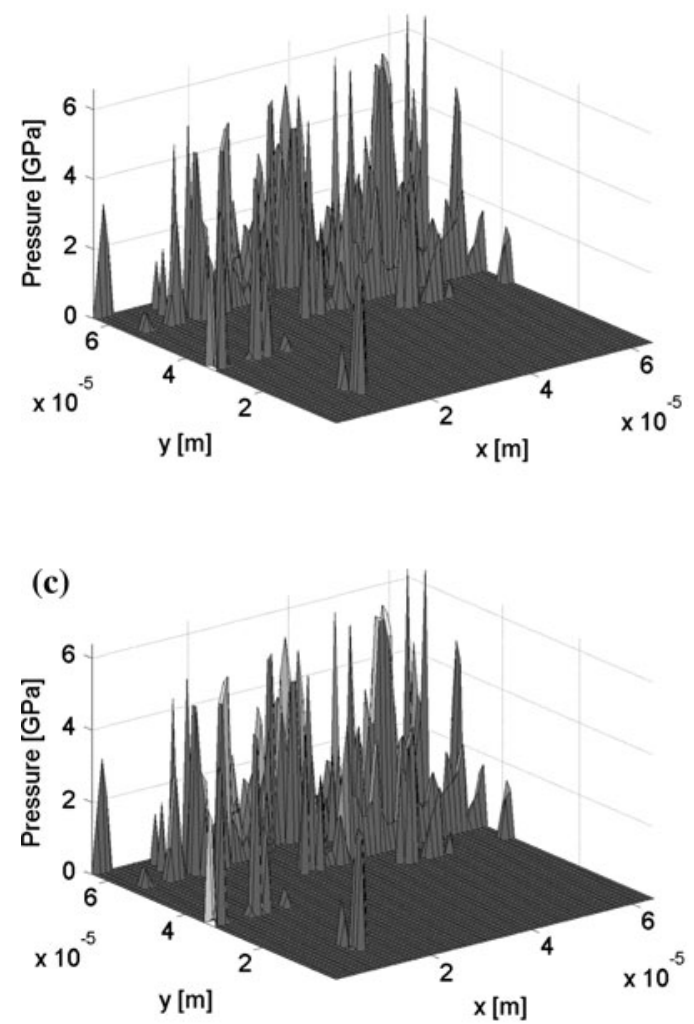

Fig. 3, the resulting stress and pressure field for the elastic calculations are shown using the input parameters given in Table 1. Here the overall coefficient of friction is set to 0.1 , which is the value used for the situation where the oil provides sufficient protection as will be discussed in the next section. The normal load is set to $0.6 \mathrm{~N}$, at this value the maximum pressure is in the range of the hardness of the bulk material and thus will give a realistic contact situation. As can be clearly seen the effect of the layer on both the stress-state in the bulk material as the normal pressure is less than $5 \%$. This justifies the simplification suggested before. In the tangent (sliding direction) the dominant influence of the chemical layer will be on the coefficient of friction. If the chemical layer is sufficient in protecting the surface the main shearing will be located in the chemical layer and thus lowering the coefficient of friction. This subject will be discussed in more detail in the next section which deals with the local nature of the coefficient of friction.

The behavior of the NC-layer can be split up into two parts: (1) elastic or (2) plastic. For the elastic part, the assumption can be made that the properties of the NC-layer
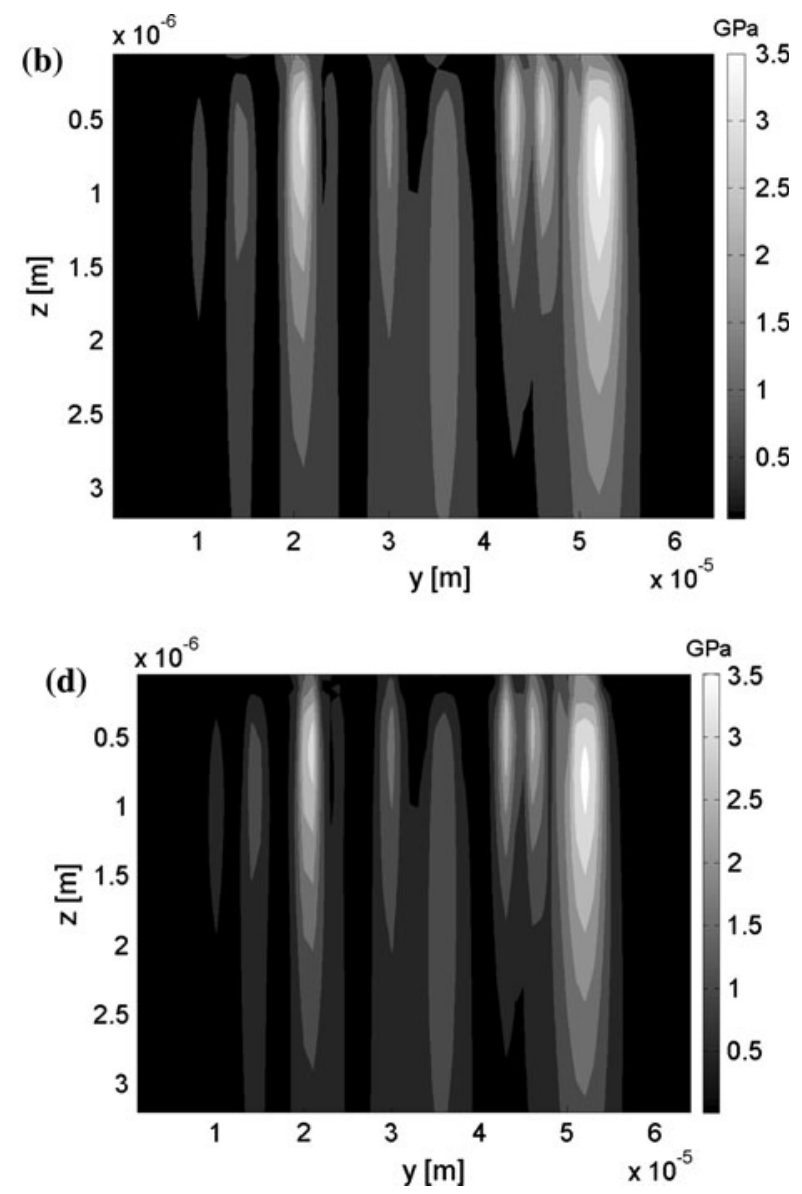

Fig. 3 Pressure profile for a coated surface $\left(p_{\max }=6.4 \mathrm{GPa}\right.$ ) and $\mathbf{c}$ uncoated surface $\left(p_{\max }=6.5 \mathrm{GPa}\right)$.Von Mises stress underneath the surface, maximum von Mises stress $\mathbf{b}$ coated material $\sigma_{v m}^{\max }=3.5 \mathrm{GPa}$ and $\mathbf{d}$ uncoated material $\sigma_{v m}^{\max }=3.55 \mathrm{GPa}$ 
with negligible porosity do not change down to a crystal size of $20 \mathrm{~nm}$, which originates from a study by Kim and Bush [24], see Fig. 4. The average grain size reported in literature for tribologically induced NC-layers at steel surfaces are in the range $20 \mathrm{~nm}$ and higher, see, e.g., [7, 9]; thus, for the elastic part a model without layers can be used for the simulations regarding steel-like materials. This enables the use of the SAM model discussed in [21] with only a small adaptation, since the plastic part is calculated separately and then imposed on the elastic bulk through the use of eigen-strains. For the exact code, the interested reader is referred to the dedicated literature about the SAM model in $[20,21,25]$, where the model is dealt with in detail. The version of the code used in this study slightly differs from the one dealt with in this literature since the convergence of the plasticity loop is over the stress rather than over the strain which is dealt with in more detail in [26]. Only a short overview of the most important parts of the code and the ones that are adapted are presented here. Starting with the reciprocal theory applied to a semi-infinite volume with boundary $\Gamma$ and volume $\Omega$. For this body, different states are defined: an initial state with internal strains $\left(u, \varepsilon, \sigma, f_{i}\right)$ and a state for the time undefined: $\left(u_{i}^{*}, \varepsilon^{*}, \sigma^{*}, f_{i}^{*}\right)$. Using the reciprocal theory and the assumption that the second state is the one in which the surface is loaded by a unit pressure $p_{i}^{*}$ at the location $A$ gives:

$u_{3}(A)=\underbrace{\int_{\Gamma_{c}} p_{i} u_{3 i}^{*}(M, A) \mathrm{d} \Gamma}_{u^{\mathrm{e}}(A)}+\underbrace{\int_{\Omega} \varepsilon_{i j}^{0}(M) C_{i j k l} \varepsilon_{i j}^{*}(M, A) \mathrm{d} \Omega}_{u^{\mathrm{P}}(A)}$

Here $u^{\mathrm{e}}(A)$ is the elastic surface displacement and by stating $\varepsilon_{i j}^{0}=\varepsilon_{i j}^{\mathrm{pl}}, u^{\mathrm{pl}}(A)$ becomes the surface displacement

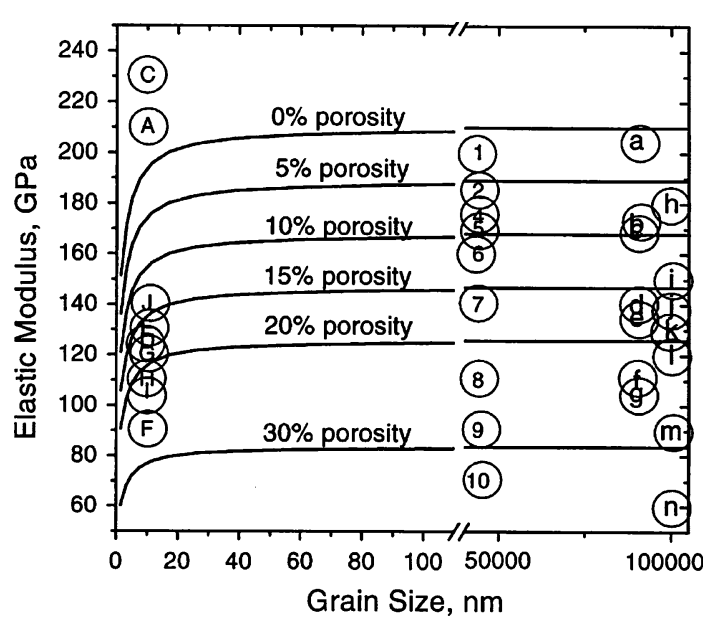

Fig. 4 Elastic modulus of steel as a function of porosity and grain size [24] due to the plastic strains inside volume $\Omega$. The surface displacement of the body can now be expressed as a function of the contact pressure and the plastic strain. To calculate the plastic strains the subsurface stresses are needed. Using the reciprocal theory again and define a state $\left(u^{* *}, \varepsilon^{* *}, \sigma^{* *}, f_{k}^{* *}\right)$, which can be seen as the state when a unit force is applied inside volume $\Omega$ at point $\mathrm{B}$. This then results in:

$u_{k}(B)=\underbrace{\int_{\Gamma} u_{k i}^{* *}(M, B) p_{i}(M) \mathrm{d} \Gamma}_{u_{k}^{\mathrm{e}}(B)}+\underbrace{\int_{\Omega} \varepsilon_{i j}^{0}(M) C_{i j k l} \varepsilon_{i j}^{* *}(M, A) \mathrm{d} \Omega}_{u_{k}^{\mathrm{p}}(B)}$

Here stating $\varepsilon_{i j}^{0}=\varepsilon_{i j}^{\mathrm{pl}}$ and only integrating over the volume where the plastic strains are not zero $\Omega=\Omega_{\mathrm{pl}}$ the displacement field is written as a function of the elastic and the plastic strains. Using Hook's law:

$$
\begin{aligned}
\sigma_{i j}^{\mathrm{tot}}(B)= & C_{i j k l}\left[\left(\frac{1}{2}\left(u_{k, l}^{\mathrm{e}}(B)+u_{l, k}^{\mathrm{e}}(B)\right)\right)\right. \\
& \left.+\left(\frac{1}{2}\left(u_{k, l}^{\mathrm{pl}}+u_{k, l}^{\mathrm{pl}}\right)-\varepsilon_{k l}^{\mathrm{pl}}\right)\right]
\end{aligned}
$$

Rewriting (3):

$\sigma_{i j}^{\mathrm{tot}}(B)=\sigma_{i j}^{\mathrm{e}}(B)+\sigma_{i j}^{\mathrm{res}}(B)$

If now the unit pressure and unit force are replaced by the complete pressure and force fields, a solution can be found for a complete rough contact situation. This is done by descrization of the surface $\Gamma$ into surface elements $N_{\mathrm{s}}$ of size $\Delta x \times \Delta y$ and the volume into elements $N_{\mathrm{v}}$ of size $\Delta x \times \Delta y \times \Delta z$. On which at the surface uniform pressures are acting and in the volume the strains inside each volume element are uniform. Starting with the elastic surface displacement which then becomes the sum of the individual pressure patches:

$u_{3}^{\mathrm{e}}(x, y)=\sum_{n=1}^{N=N_{\mathrm{s}}} D_{3 i}^{n}\left(x-x_{n}^{\prime}, y-y_{n}^{\prime}\right) p_{i}^{n}\left(x_{n}^{\prime}, y_{n}^{\prime}\right)$

Here $x, y$ are the coordinates of observation and $x_{n}^{\prime}, y_{n}^{\prime}$ are the coordinates of the center of the excitation patch $n$, the expressions of $D_{3 i}^{n}$ are given in the appendix, where $i$ can have the value of 3 for the displacement due to normal force and 1 for the displacement due to traction. Next is the displacement due to the plastic strains $\varepsilon_{i j}^{\mathrm{p}}$. Using the assumption of uniform strains within the volume elements:

$u_{3}^{\mathrm{pl}}(x, y)=\sum_{n=1}^{N=N_{v}} K_{i j}^{\mathrm{pl}}\left(x-x_{n}^{\prime}, y-y_{n}^{\prime}, z_{n}^{\prime}\right) \varepsilon_{i j}^{\mathrm{p}}\left(x_{n}^{\prime}, y_{n}^{\prime}, z_{n}^{\prime}\right)$

Here $(x, y)$ is the location of the point of observation on the surface and $\left(x_{n}^{\prime}, y_{n}^{\prime}, z_{n}^{\prime}\right)$ are the coordinates of the excitation volume $n$. The expression for $K_{3 i}^{\mathrm{pl}}$ is given in the appendix. 
The stresses for the total system, given in Eq. 4, can also be expressed in a summation form:

$$
\begin{gathered}
\sigma_{i j}^{\mathrm{tot}}(x, y, z)=\sum_{n=1}^{N=N_{\mathrm{s}}} S_{i j k}^{\mathrm{elas}}\left(x-x_{n}^{\prime}, y-y_{n}^{\prime}, z-z_{n}^{\prime}\right) p_{k} \\
+\sum_{n=1}^{N=N_{v}} S_{i j}^{\mathrm{pl}}\left(\left(x-x_{n}^{\prime}, y-y_{n}^{\prime}, z-z_{n}^{\prime}, z+z_{n}^{\prime}\right)\right. \\
\left.\quad \times \varepsilon_{i j}^{\mathrm{pl}}\left(x_{n}^{\prime}, y_{n}^{\prime}, z_{n}^{\prime}\right)\right)
\end{gathered}
$$

Here the expressions for $S_{i j}^{\mathrm{elas}}$ and $S_{i j}^{\mathrm{pl}}$ for the different parts are given in the appendix, where the expression originally used in the code discussed in [20,21] are replaced by more efficient equations originating from [27].

Now the surface displacement and the stress inside the bulk material of an elastic half space with plastic strains inside the volume are described by equations it can be used to model the contact of the elasto-plastic contact between two half spaces using the model depicted Fig. 5 of which the flow chart is shown in Fig. 6.

One part will be discussed here in detail with respect to the plastic loop and the validation of the code. In the original code the Prandtl Reuss method is used to compute the plastic strains this is later on adapted by Nelias et al. [21] to a return mapping algorithm to increase the efficiency of the code.

The current model also uses a return mapping algorithm, however, in the current version the plastic loop is changed to a stress related one rather than strain. As can be seen in [20] the plasticity loop is stopped if convergence of the plastic strain is reached. However, the basis of the plasticity theory states that for a system to stay in the elastic regime the elastic stresses should be on the yield surface:

$\sigma_{i j} \leq \phi$

This is, however, not per definition satisfied if the convergence criterion used is based on the plastic strain inside the body. In the current model, the plasticity loop used is depicted in Fig. 7. Here the yield surface is defined by the von Mises yield criterion and the loop is stopped if the stress state in the complete meshed volume is either within the yield surface or within the predefined error outside the yield surface. Also the iteration on the stress rather than on the strain ensures that the plasticity loop stays stable, since the return mapping algorithm is unconditionally stable. To validate the new plasticity loop the examples given in Fig. 5 [21] are reproduced and the results are presented in Fig. 8. It can be concluded that the current code gives approximately the same results for the low-friction situation and slightly better results for the high-friction situation. However, the results of the SAM code will not correspond completely with the FEM solution since the tangential displacement is taken into account in a decoupled method in the SAM code while in the FEM code they are coupled. Now the complete surface
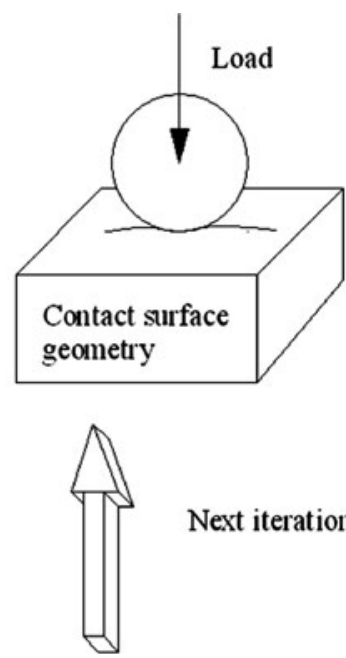

Next iteration

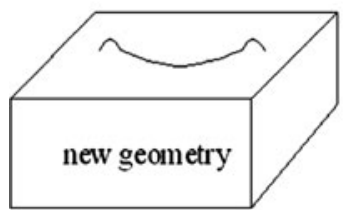

Displacement superposition

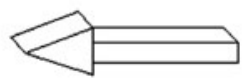

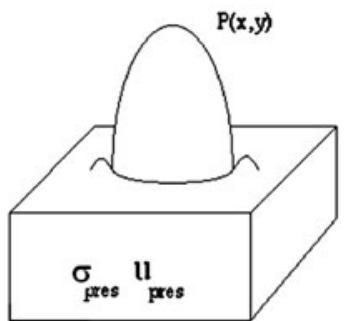

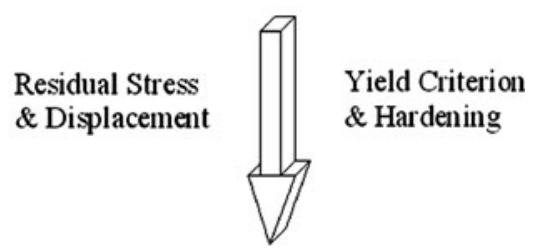

Fig. 5 The iterative process of solving the elasto-plastic contact 


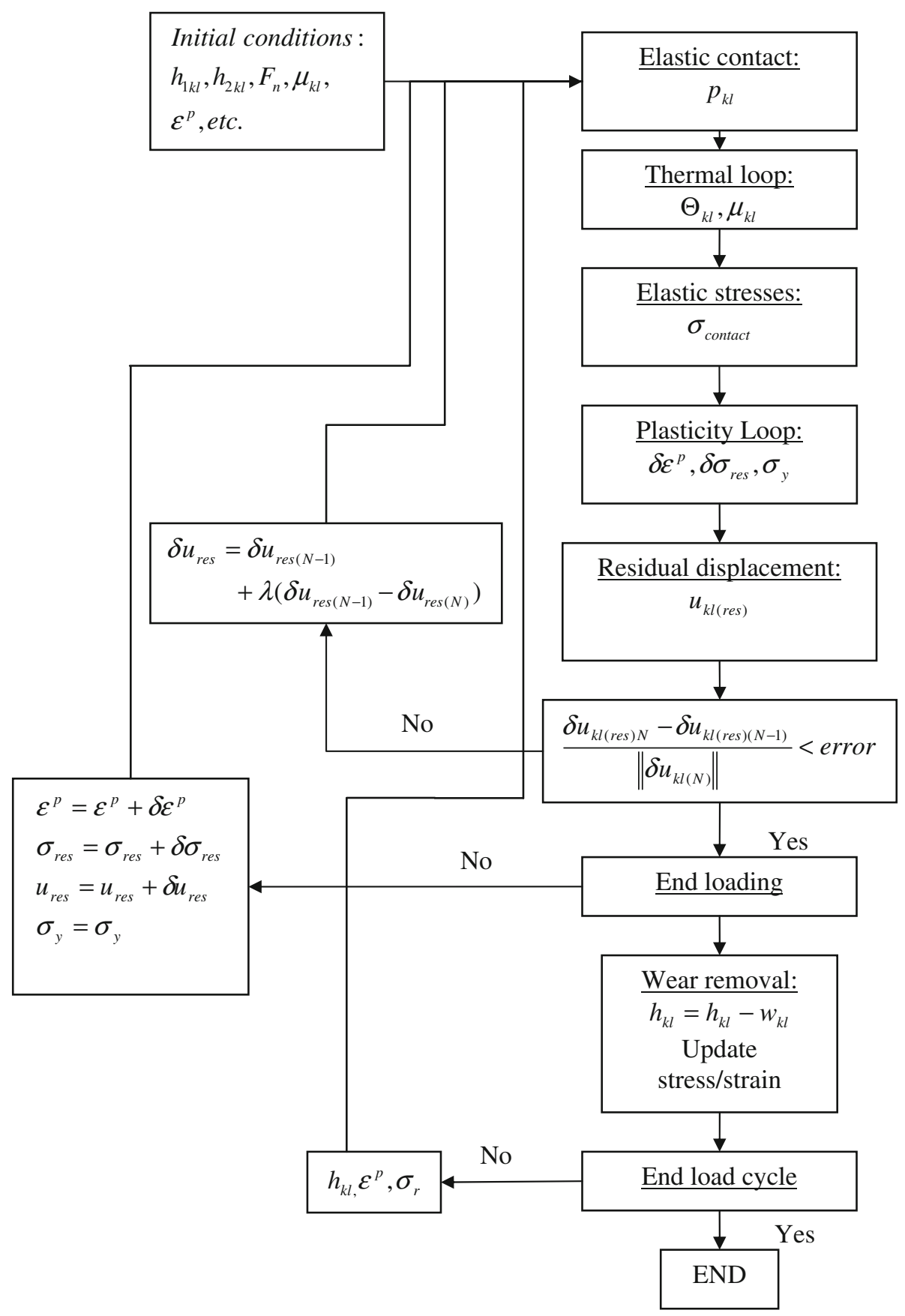

Fig. 6 Flowchart of wear model

displacement and stress state can be calculated and the contact problem stated as:

$$
\begin{aligned}
& h(x, y)=h(x, y)+\delta+u_{3}^{(1+2)}(x, y) \\
& h(x, y) \geq 0 \quad \text { then } \quad p(x, y) \geq 0 \\
& h(x, y)<0 \quad \text { then } \quad p(x, y)=0
\end{aligned}
$$

The inequalities stated by Eq. 9 can be solved using the CGM method [28]:

$$
\begin{aligned}
p_{k+1} & =p_{k}-\frac{r_{k}^{T} r_{k}}{d_{k}^{T} D_{3 i}^{n} d_{k}} d_{k} \\
r_{k+1} & =r_{k}-\frac{r_{k}^{T} r_{k}}{d_{k}^{T} D_{3 i}^{n} d_{k}} D_{3 i}^{n} \\
d_{k+1} & =-r_{k+1}+\frac{r_{k+1}^{T} r_{k+1}}{r_{k}^{T} r_{k}}
\end{aligned}
$$

The above method is the elastic loop in Fig. 6. 


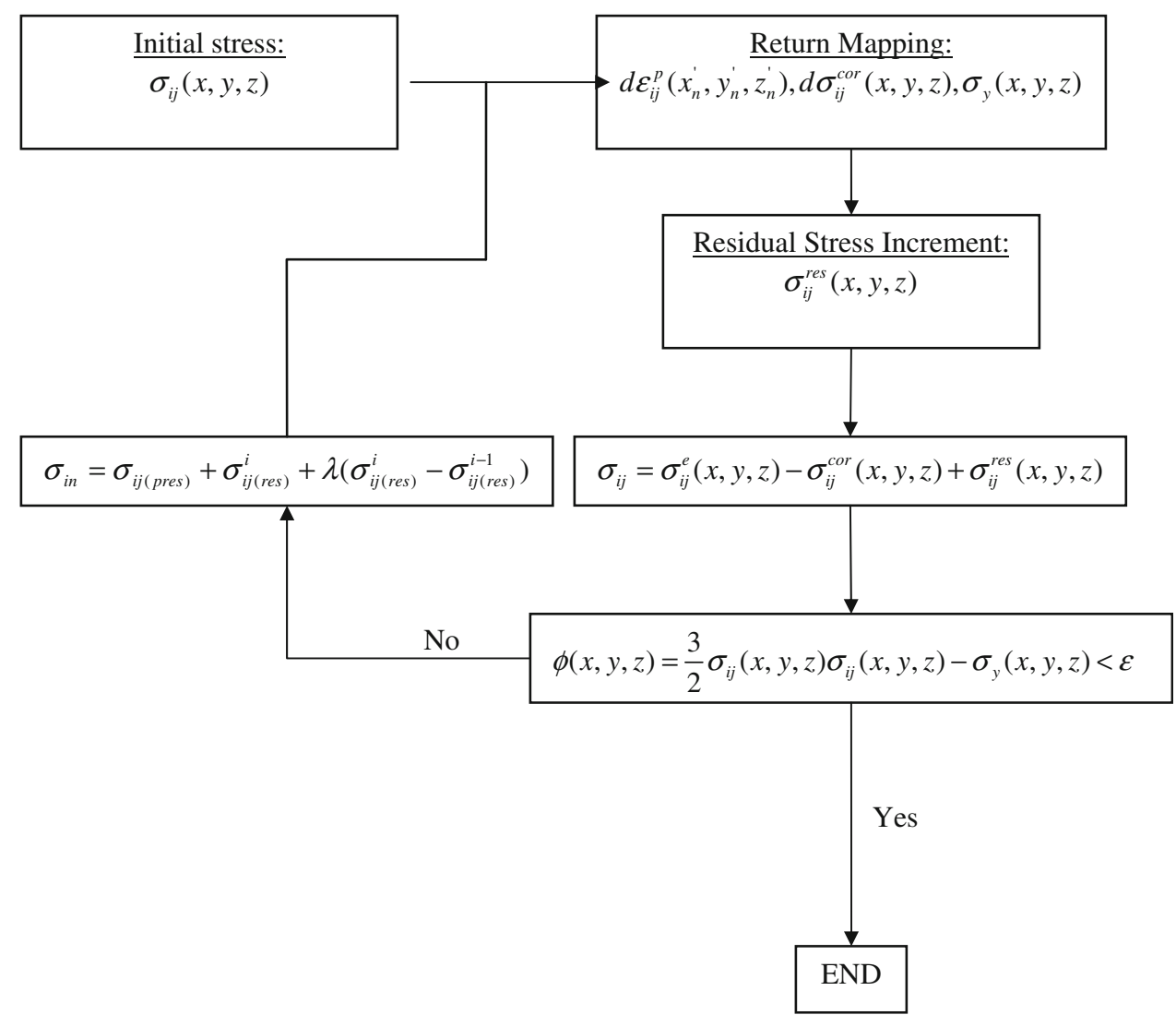

Fig. 7 New "plasticity loop" based on the stress relaxation rather than on the relaxation of the plastic strain

\section{Material Model}

The NC-layer is included in the contact model using a different model for the yield stress and hardening behavior for the fist few elements in depth direction. However, the exact value of the plastic properties of the first few hundred nanometers underneath the surface is currently still not fully defined due to lack of understanding of the NC-layer. In literature, the different authors have different opinions since different measured values are found for the hardness of the materials near the surface as a result of crystalline changes due to tribological/mechanical interaction or artificially created NC-layers (see for example [10-12, 2326]). Therefore, in this article the exact numerical data will not be the point of particular interest or will the origin of the layer be. The focus will be on the effect the different yield behaviors have on the wear modeling. This approach gives the opportunity to make a clear distinguishing between the two main theories which both will be dealt with. The first one is that the NC-layer only exhibits hardening: introducing a hard surface layer capable of withstanding the high local pressures present at the peaks of the asperities, resulting in a hardness profile shown in Fig. 9a. The second theory states that the NC-layer formed has a gradual increase/decrease curve and thus has a very ductile nature.
This will prevent high shear strains in the bulk material preventing the forming of cracks in the bulk material while the NC-layer accommodates the required plastic strain. The hardness profiles resulting from this theory are presented in Fig. 9b. These curves are reproduced from measurements presented in [6]. The three curves represented: curve A originates from a new sampled after polishing, curve B from a mild wear test, and curve $\mathrm{C}$ from a failed (high wearing) system. These three curves are mainly of interest to see if the change seen from hardness profile A to curve B has a positive effect in the simulations, since this is the profile created and thus favored by a system with low wear.

\section{Friction Model}

The next step is to formulate a criterion for the failure of the boundary layers so the local coefficient of friction can be determined. The coefficient of friction will be split into two regimes: high local coefficient of friction by metal-tometal contact and a low coefficient of friction where the main shearing is located in the chemical reaction layer. The defined regimes have different coefficients of friction in the range of 0.1 and 0.4 for protected and unprotected areas, respectively. The transition from the one to the other 

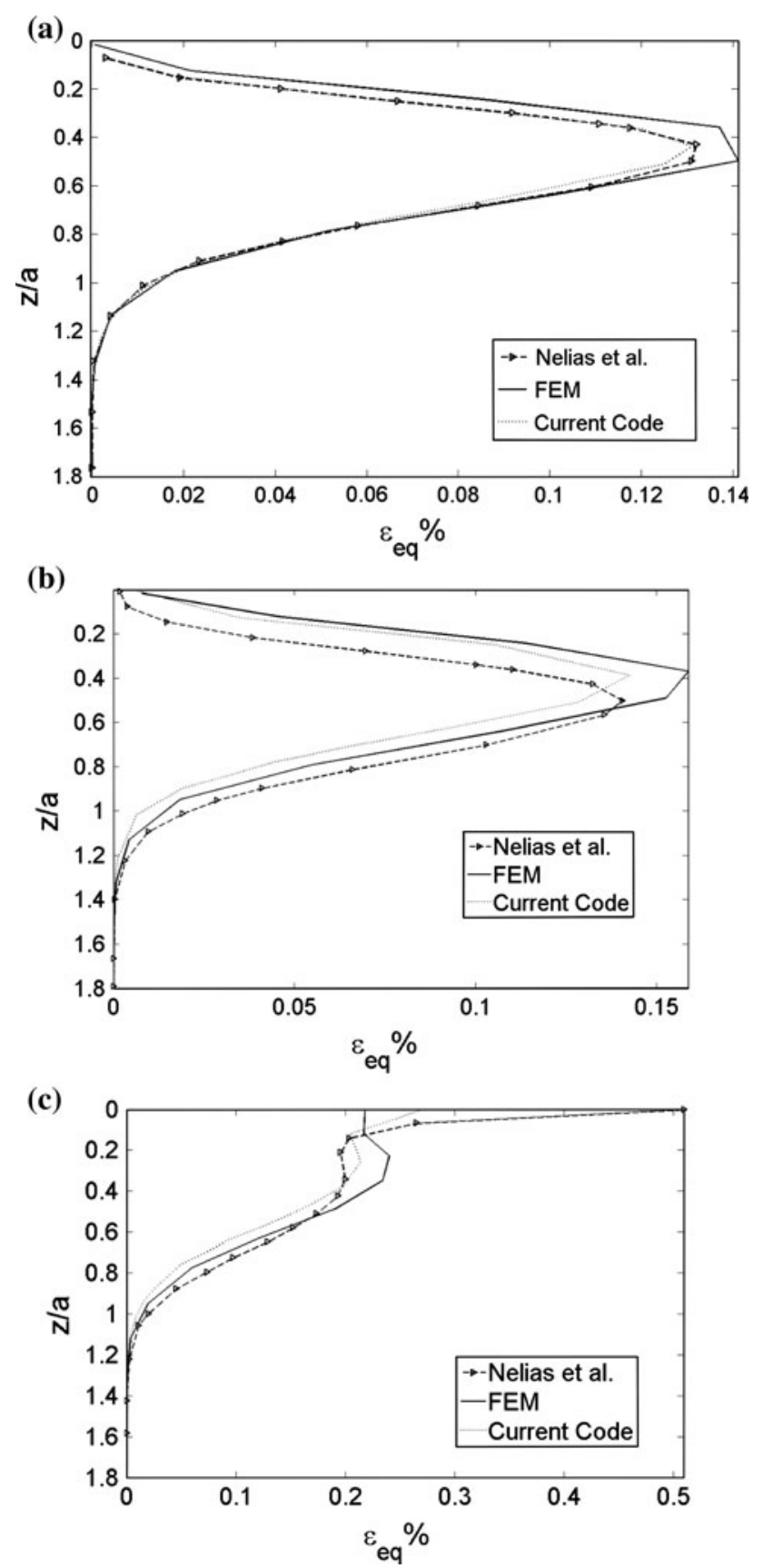

Fig. 8 Equivalent plastic strain for the elasto-plastic solutions given in [21] compared with the current code and FEM results a for the frictionless case $\mathbf{b}$ cof $=0.2 \mathbf{c}$ cof $=0.4$

is seen as a stepwise phenomenon, if the failure criterion of the layer is transcended the coefficient of friction "jumps" to the higher value. A representation of the boundary layer is given in Fig. 1. Here the adsorbed layer is equivalent to the high viscous layer reported in different studies and does not play a role in the anti-wear properties at severe conditions [29-31], which are typical for run-in situations. To model the chemically reaction layer an assumption has to be made regarding the material model to be used for this (a)

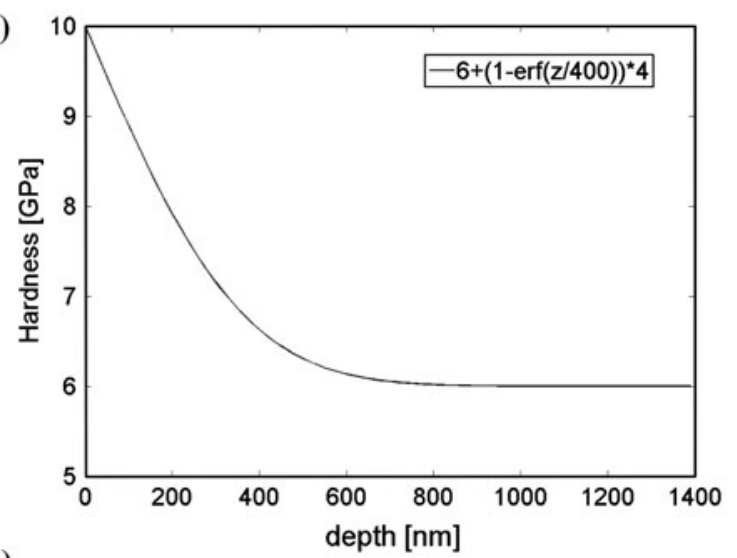

(b)

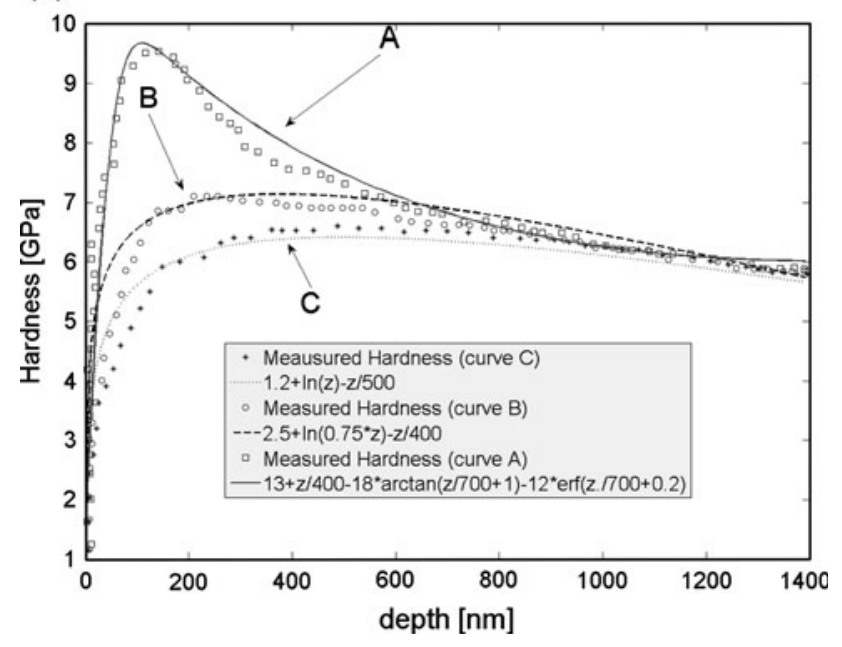

Fig. 9 Hardness profile according to measurements presented in literature. a Increasing hardness toward the surface. b Different hardness profiles for curve A polished surface, curve B mild wearing surface, and curve $\mathrm{C}$ for a severe wearing surface [7]

layer, e.g., what kind of behavior this layer has. Based on the study presented in [32] it is suggested that the chemical reacted boundary layers acts as a solid rather than a viscous fluid. In this study the boundary layer is thus modeled as an elastic-plastic solid, where the transition from elastic to plastic is defined by the von Mises yield criterion as is used for the bulk material. The properties of this layer are based on averaging the properties given from the different studies are presented in Table 2. The main properties determined from this table are the thickness of the layer estimated at $100 \mathrm{~nm}$ and the Young's modulus of the layer of $80 \mathrm{GPa}$.

The yield stress of the surface layer is not very well defined in the different studies and ranges from 2 to $6 \mathrm{GPa}$. However, if the layer thickness is estimated at $100 \mathrm{~nm}$ the indentation depth is not to exceed $10 \mathrm{~nm}$ for the hardness measurement. This is not the case for the measurements reporting $6 \mathrm{GPa}$ and to the authors opinion the hardness of the NC-layer/bulk material is influencing the measurement significantly and this results in an unrealistic high hardness. 
Table 2 Results for thickness and Young's modulus for the chemical reacted layer retrieved from literature resulting from rubbing experiments in ZDDP-rich oils

\begin{tabular}{lllrll}
\hline References & $P_{n}(\mathrm{MPa})$ & $V(\mathrm{~m} / \mathrm{s})$ & $T_{\text {bath }}\left({ }^{\circ} \mathrm{C}\right)$ & $\begin{array}{l}t_{\text {layer }} \\
(\mathrm{nm})\end{array}$ & $\begin{array}{l}E_{\text {layer }} \\
(\mathrm{GPa})\end{array}$ \\
\hline$[39]$ & 504 & 0.3 & 100 & 115 & $85-75$ \\
{$[29]$} & 504 & 0.3 & 100 & $60-120$ & - \\
{$[30]$} & 700 & 0.03 & 83 & $40-100$ & - \\
{$[31]$} & 700 & - & 83 & 100 & - \\
{$[40]$} & 500 & $\sim 0.3$ & 100 & $30-60$ & - \\
{$[41]$} & - & - & - & 100 & 81 \\
{$[42]$} & 400 & $\sim 0.3$ & 100 & 70 & 96 \\
{$[43]$} & 500 & $\sim 0.3$ & 100 & 300 & $120-90$ \\
{$[44]$} & $\sim 300-500$ & 0.03 & 83 & $<100$ & 130 \\
{$[45]$} & $10-50$ & $0.25-0.55$ & 100 & $<100$ & \\
{$[46]$} & 360 & - & 100 & 140 & 90 \\
{$[47]$} & $\sim 425$ & 0.34 & 100 & $160>$ & 122.7 \\
{$[48]$} & 590 & $\sim 0.3$ & 100 & $\sim 160$ & 81 \\
{$[49]$} & 600 & 0.01 & 80 & $<60$ & - \\
{$[50]$} & 135 & $\sim 0.35$ & 100 & $60-180$ & $90-120$ \\
{$[51]$} & 300 & $\sim 0.3$ & 100 & $30-60$ & - \\
{$[52]$} & 950 & 0.1 & 100 & 120 & - \\
\hline & & & & & \\
\hline
\end{tabular}

For this reason the simulations are done with a chemical layer with a hardness of $2 \mathrm{GPa}$, which results in a yield stress of approximately $0.7 \mathrm{GPa}$.

The model described before can visually be expressed as show in Fig. 10, where the bulk material is protected by "friction-elements," representing the chemical layer, which currently have the sole purpose to indicate the coefficient of friction locally present, under which the NC material is located. The pressure criterion is based on the idea first presented in [33,34], where it is suggested the growth of tribo-chemical film protecting the surface must be greater then the removal rate for the lubricant to protect the surface against severe wear. Here it is assumed that the growth is diffusion-based process and can be expressed as:

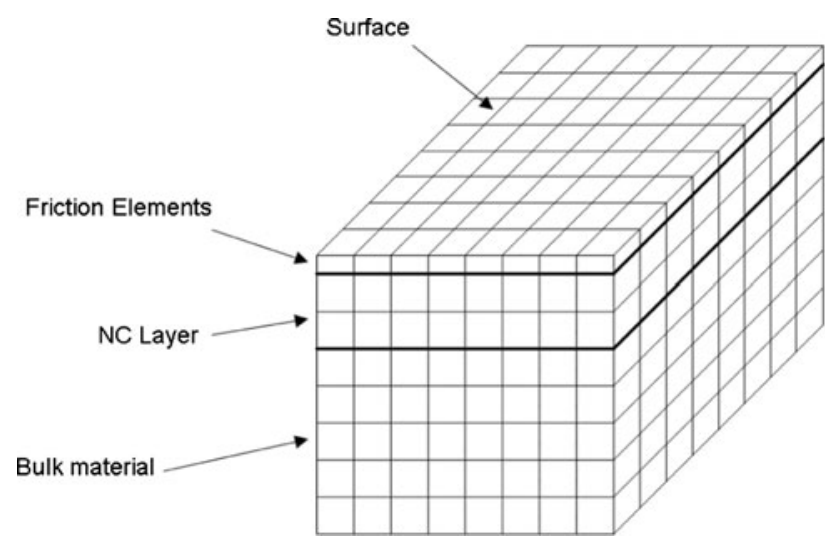

Fig. 10 Representation of the wear model built up
Table 3 Effective diffusion coefficients measured in [33] at different oil bath temperatures at nominal contact pressures of $22 \mathrm{MPa}$

\begin{tabular}{ll}
\hline $\begin{array}{l}\text { Oil bath } \\
\text { temperature } T\left({ }^{\circ} \mathrm{C}\right)\end{array}$ & $\begin{array}{l}\text { Eff. diffusion } \\
\text { coefficient } K_{0}\left(\mathrm{nms}^{-1 / 2}\right)\end{array}$ \\
\hline 50 & 2.451 \\
100 & 3.644 \\
150 & 4.620 \\
200 & 4.916 \\
\hline & \\
&
\end{tabular}

the different values of $K_{0}$ are given in Table 3 .

The nominal pressure is lower than the system studied, however, since it is assumed that the growth of the layer will take place outside the contact area the main factor influencing the growth rate will be the temperature of the oil.

The amount the chemical layer removal is indicated by the plastic strain in the direction normal to the surface $\left(\varepsilon_{z z}^{\text {layer }}\right)$. Due to its limited thickness it is safe to assume all stresses do not vary through the thickness of the layer $\left(\frac{\partial \sigma_{i j}}{\partial z}=0\right)$, e.g., the plain stress state. To calculate the plastic strain, the following simplification of the conditions the layer has to withstand are made. From the bulk side the layer is stretched by the strain of the bulk material, because it sticks to the bulk material (no slip condition between layer and bulk material) and at the top the pressure and shear is put on the surface of the layer. This results for the pre-strain in:

$$
\begin{aligned}
\sigma_{i i_{\text {layer }}}= & \frac{E_{\text {layer }}}{2\left(1+v_{\text {layer }}\right)} \varepsilon_{i i_{\text {bulk }}} \\
& +\frac{v_{\text {layer }} E_{\text {layer }}}{\left(1+v_{\text {layer }}\right)\left(1-2 v_{\text {layer }}\right)}\left(\varepsilon_{i i_{\text {bulk }}}+\varepsilon_{j j_{\text {bulk }}}+\varepsilon_{k k_{\text {bulk }}}\right)
\end{aligned}
$$

where $i, j$, and $k$ can be $x, y$, or $z$ and the external pressures from the top side are given by the normal load and the traction:

$\sigma_{z z_{\text {layer }}}=\sigma_{z z_{\text {layer }}}+P$

$\sigma_{x z_{\text {layer }}}=\mu P$

Using these stress conditions the plastic strain can be calculated. The maximum plastic strain allowed in normal direction is determined by the amount of growth gained every cycle. This is done to satisfy the chemical balance which is needed for the protection:

$\dot{X}(t) \geq \dot{W}(t)$

Here $\dot{X}(t)$ is the growth rate of the chemical film and $\dot{W}(t)$ is the wear rate. If now the growth rate is expressed per 


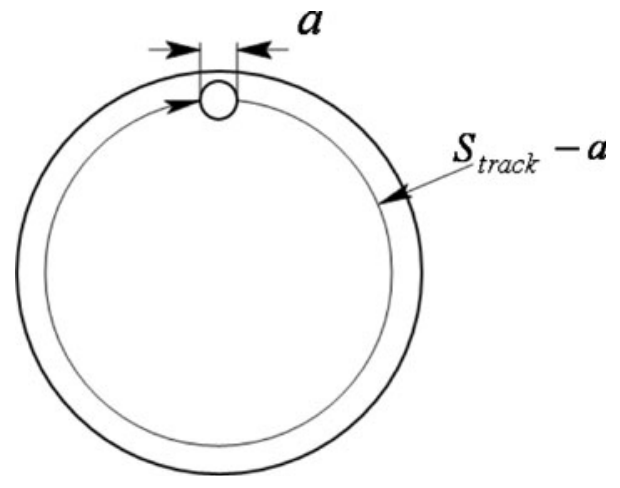

Fig. 11 Wear track versus apparent contact area

cycle and it is assumed the layer only grows outside the apparent contact area the time of growth will be, see Fig. 11:

$t_{\mathrm{gr}}=\frac{s_{\text {track }}-a}{\left|V_{1}-V_{2}\right|}$

The maximum amount of plastic strain allowed in the normal direction over the thickness of the layer per contact cycle can be expressed as:

$\varepsilon_{z z \max }^{\text {layer }}=\frac{\sqrt{t_{\mathrm{gr}}} K_{0}}{h_{\text {balance }}}$

Here $h_{\text {balance }}$ is the layer thickness measured for a system, see Table 2.

\section{Wear Model}

In the current model, the coefficient of friction is determined locally as discussed in the previous section by assuming the protection of the lubricant is limited to preset contact conditions, e.g., a maximum amount of chemical film that is allowed to be removed each contact cycle given by Eq. 17. As for the coefficient of friction also the wear can be thought of as a 2-fold mechanism: local severe wear and local mild wear. For the mild wear (protected) situation it is first assumed that negligible wear will occur at these locations since the shearing will be located in the boundary layers formed on the steel surface and thus no bulk material will directly be removed. This corresponds well with real systems running in mild conditions since particles generated by this type of systems are mainly built up from chemical products which originated from the chemicals present in the oil $[34,35]$.

The severe wear is based on the brittle wear behavior of high-strength carbon steels and is associated with a high coefficient of friction. The train of thought in the wear model used here is introduced by Nelias et al. [19]. The wear model is found upon a recent study of Oila and Bull
[35], who stated that micro-pitting was related to microcracks underneath the surface. To predict the formation of these micro-cracks a crack criterion is needed, which is currently simulated as a maximum equivalent plastic strain. It is then assumed that the crack runs along the line of the plastic volume both reaching the surface and transcending the critical strain value, creating a wear volume. In the current simulations, it is assumed that the wear volume is removed in only one cycle directly after it is formed. Incorporating this threshold directly in the system creates very large wear since sharp edges will be created on the sides of the worn volume. To deal with this problem the volume removed is smoothened using a Savitzky-Golay smoothing filter. This, however, introduces numerical artifacts, which can be used to modify the model for less or more ductile materials. This is left out in the discussion in [19] as will be done currently for brevity, but is discussed in detail in [36] and the reader is thus referred to this study for more details on the smoothening. The complete wear model is schematically shown in Fig. 6. To reduce the effect of the element size on the wear volume an interpolation is used to determine the wear depth, again for more details the reader is referred to [36].

\section{Results}

Using the methods discussed above a set of examples will be calculated, using a relatively rough surface of a hard turned pin (Fig. 12). This surface is measured using an inter-reference microscope with a lateral resolution of $1 \mu \mathrm{m}$ and a depth resolution of $1 \mathrm{~nm}$. The $R a$ value of the surface is $0.270 \mu \mathrm{m}$. Using the values of $K_{0}$ given in Table 3, a contact width of $300 \mu \mathrm{m}$, total wear track of $314.15 \mathrm{~mm}$ (e.g., track radius of $50 \mathrm{~mm}$ ), a oil bath temperature of $100{ }^{\circ} \mathrm{C}$ and a sliding speed of $1 \mathrm{~m} / \mathrm{s}$ gives a

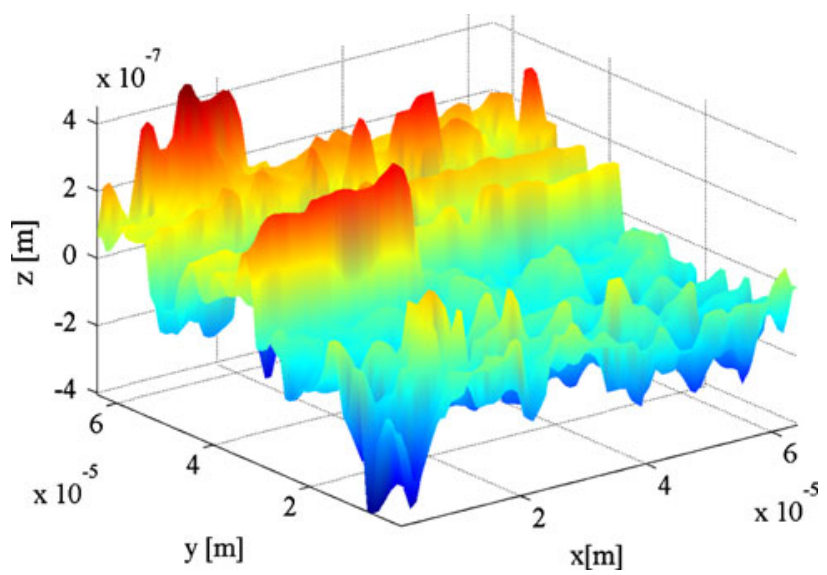

Fig. 12 Surface profile of the surface used in the simulations: a relatively rough, hard turned surface 
growth of the chemical layer of $2.04 \mathrm{~nm}$ for each cycle. Using an average chemical layer thickness of $100 \mathrm{~nm}$ results in $\varepsilon_{z z \max }^{\text {layer }}=2 \%$, which will be used in the current simulations. The nominal contact pressure for the contact simulations is kept at approximately $150 \mathrm{MPa}$ (a normal load of maximal $0.6 \mathrm{~N}$ ). This load is applied in a linear increasing and decreasing signal while a Coulomb friction law is used to simulate a quasi-sliding situation. A load

(a)
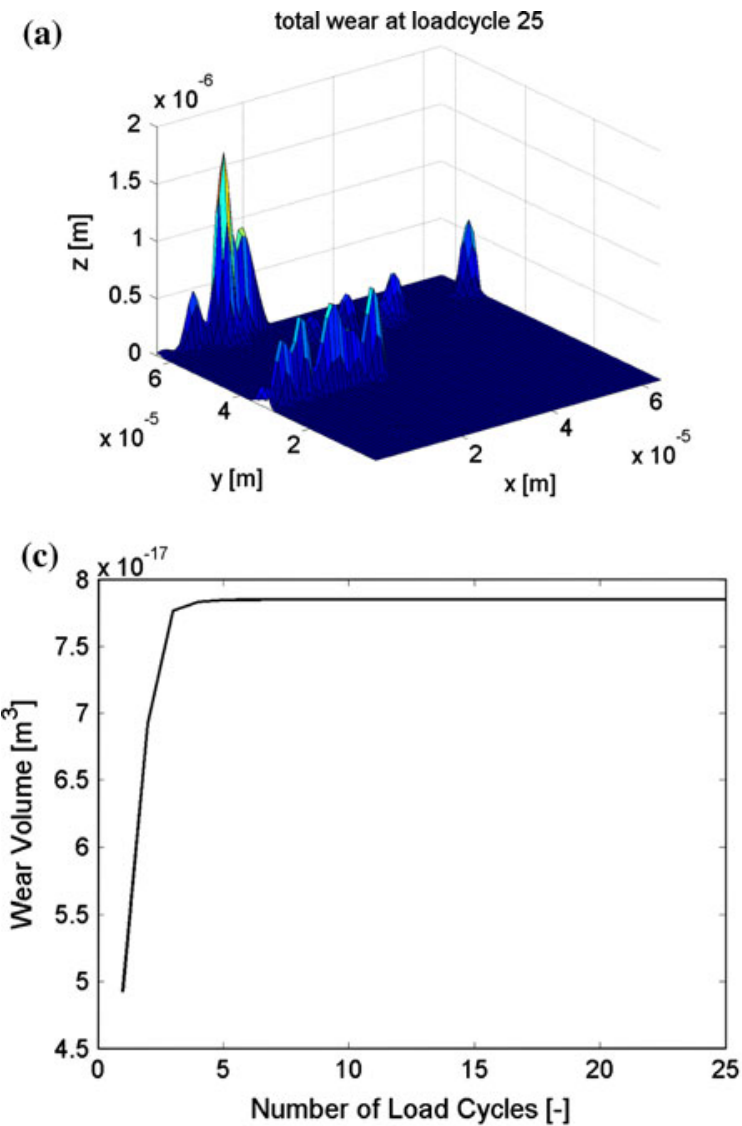

(e)

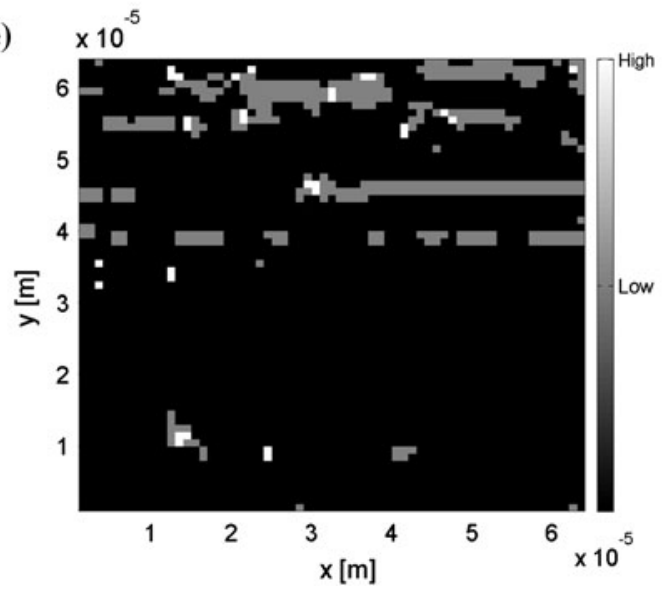

Fig. 13 Results for the simulation with bulk material properties a wear volume after 25 cycles. b Original surface geometry (transparent) versus worn surface (solid). c Wear volume as a cycle refers to a complete increase and decrease of the normal load while a load step refers to one incremental load step.

The next step is setting up a threshold for the equivalent plastic strain at which the bulk material and $\mathrm{NC}$ material will tear. Here two different material models will be evaluated, one for the base material and one for the NC-layer. The base material is represented by a
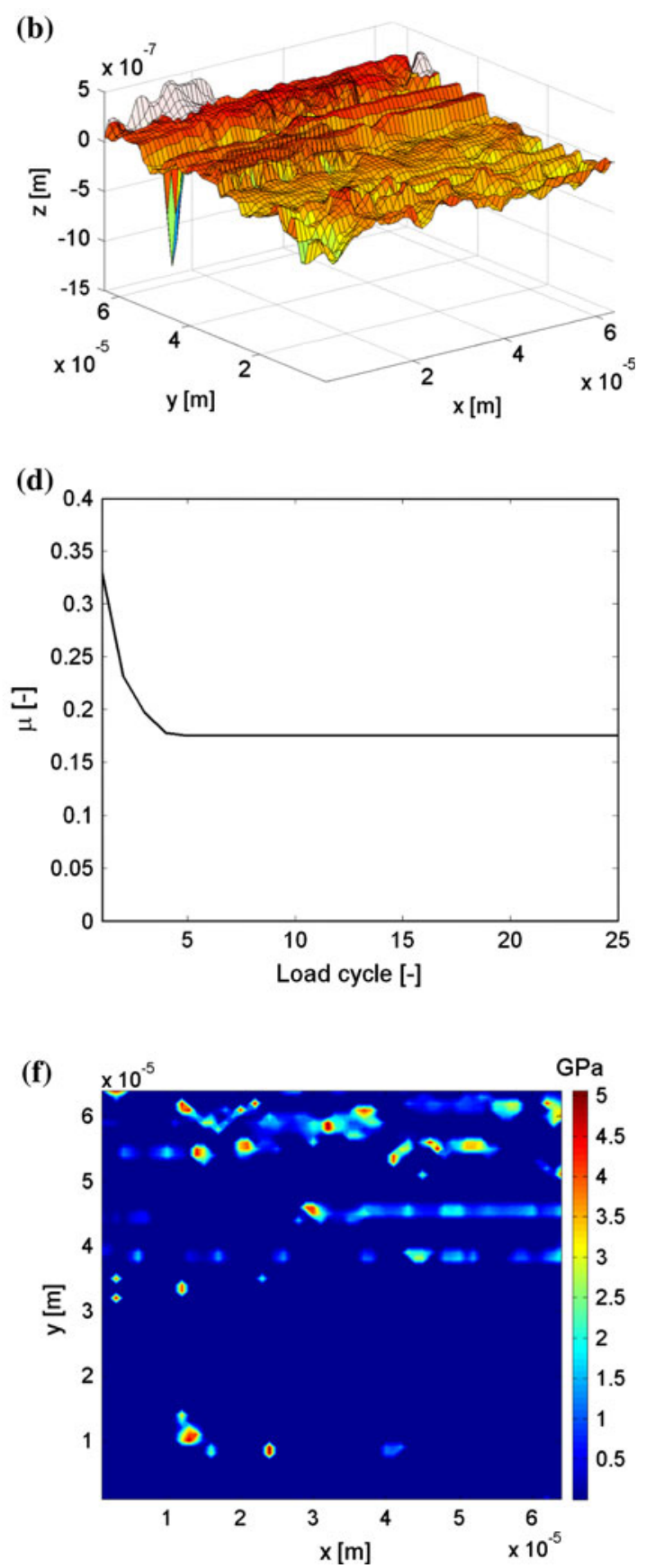

function of load cycle. d Coefficient of friction versus load cycle. e Local coefficient of friction at cycle 25 (black no contact, white high friction, gray low friction. f Pressure field 
failure strain given in [19] of $0.2 \%$ equivalent strain. For the NC-layer, a higher equivalent strain will be used in the range of $2 \%$. In reality, this value might be much higher since in literature super plasticity is reported for some types of $\mathrm{NC}$ material. The reason for the adaptation of the material model is to give show the effect of a more ductile soft layer at the surface as would be formed by the tribosystem through the reduction of the grain size in the form of a NC-layer underneath the surface [8, 37]. For the work hardening of both the materials a Swift hardening law combined with an isotropic hardening model is used: (a)

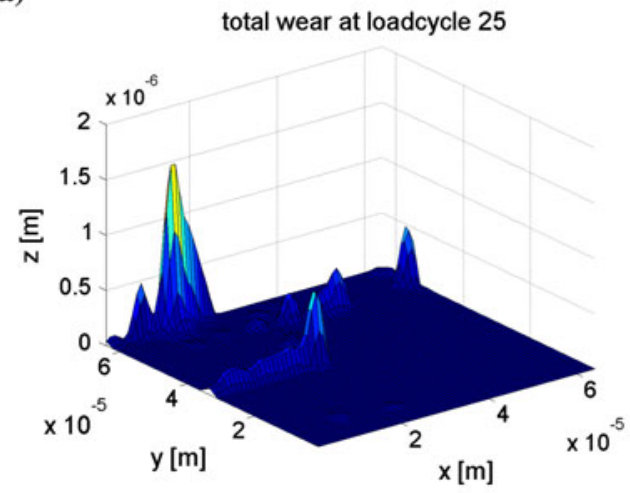

(c)

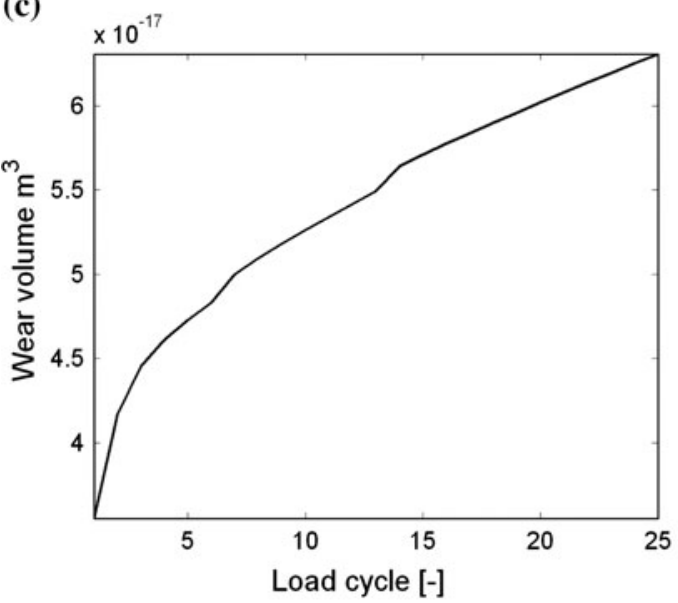

(e)

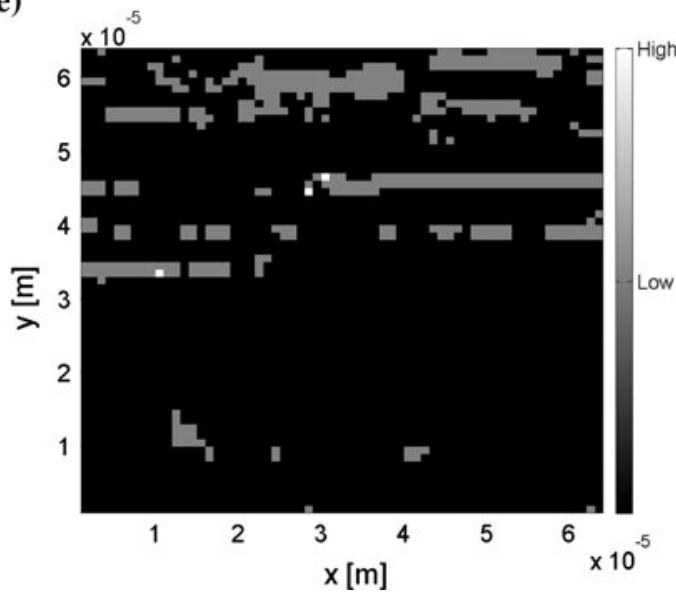

Fig. 14 Results for the simulation with material curve A: a wear volume after 25 cycles. b Original surface geometry (transparent) versus worn surface (solid). c Wear volume as a function of load (b)
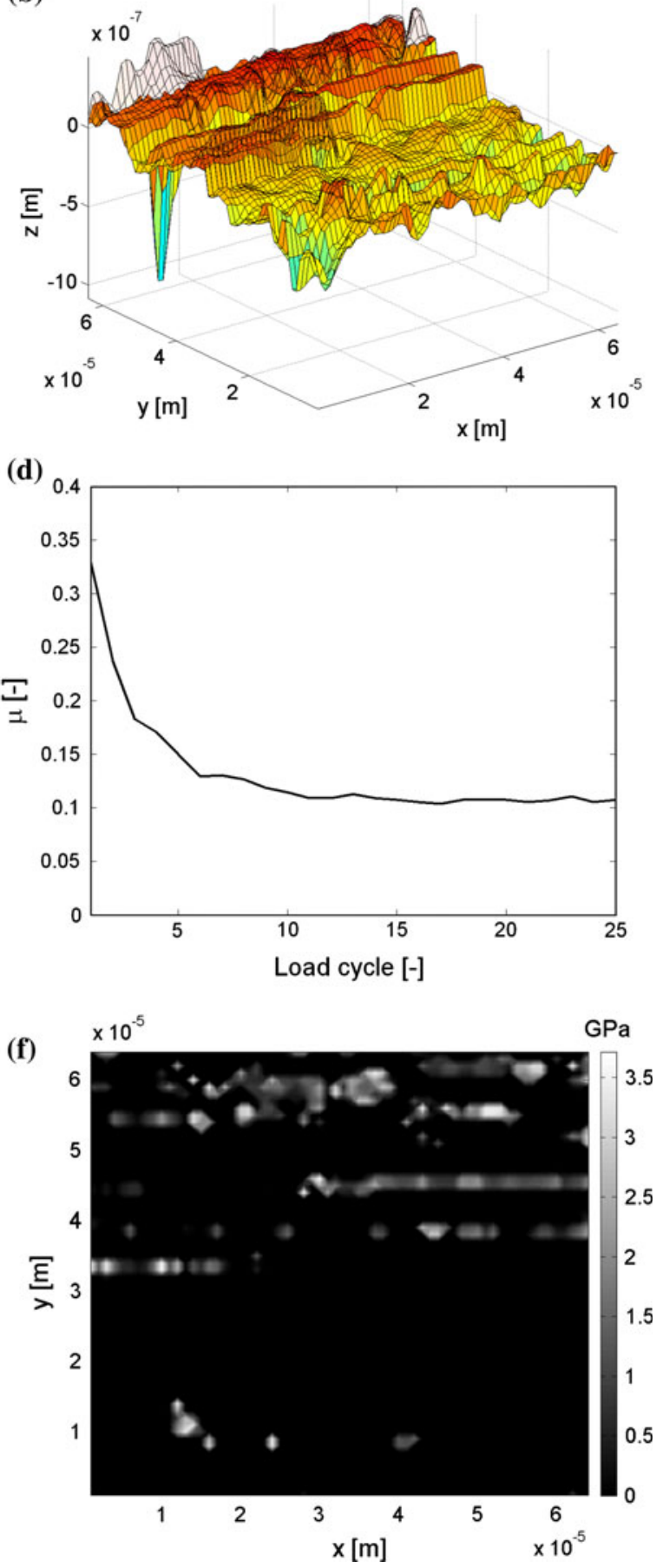

cycle. d Coefficient of friction versus load cycle. e Local coefficient of friction at cycle 25 (black no contact, white high friction, gray low friction. f Pressure field 
$\sigma_{\text {yield }}\left(\varepsilon_{\mathrm{pl}_{\mathrm{eq}}}\right)=B\left(C+\varepsilon_{\mathrm{pl}_{\mathrm{eq}}} \cdot \alpha\right)^{n}$

The parameters used are $B=1780 \mathrm{MPa}, C=4$, and $n=0.095$. If $\alpha$ is set to $10^{6}$ the material model represents AISI 52100, which is a highly work hardening material with very limited plastic strain $\varepsilon_{\mathrm{pl}} \mathrm{eq}_{\max }=0.2 \%$.

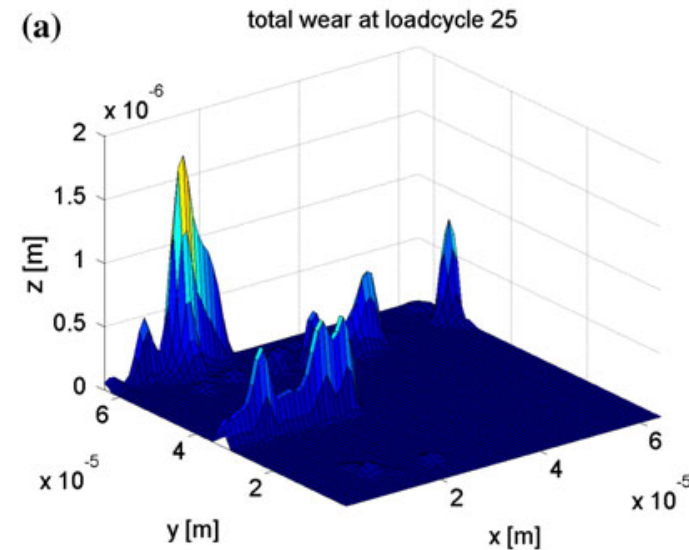

(c)
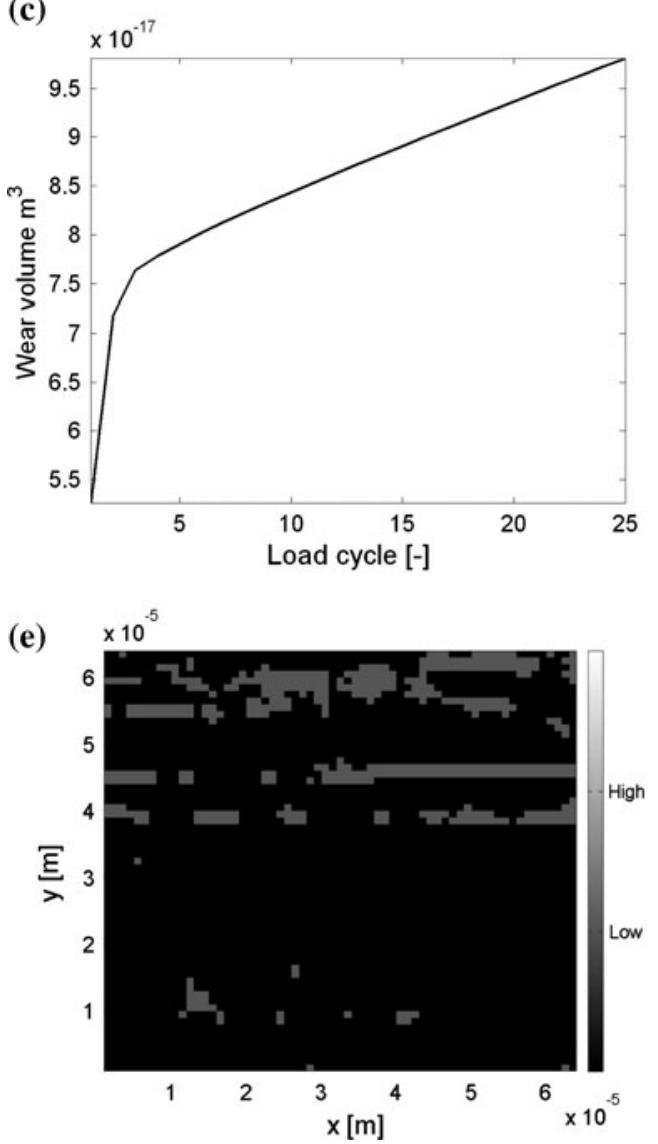

Fig. 15 Results for the simulation with material curve B/C. a Wear volume after 25 cycles. b Original surface geometry (transparent) versus worn surface (solid). c Wear volume as a function of load

\section{Simulation Results}

For the brittle bulk material model the results are presented in Fig. 13. Here it can be seen that the mean wear takes place in the first six load cycles and then is reduced to only mild wear, see Fig. 13c, d. This suggests very good wear

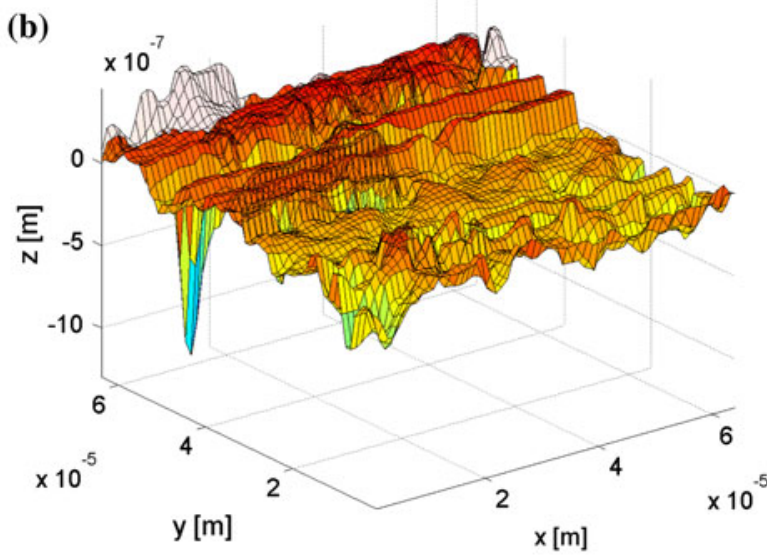

(d)

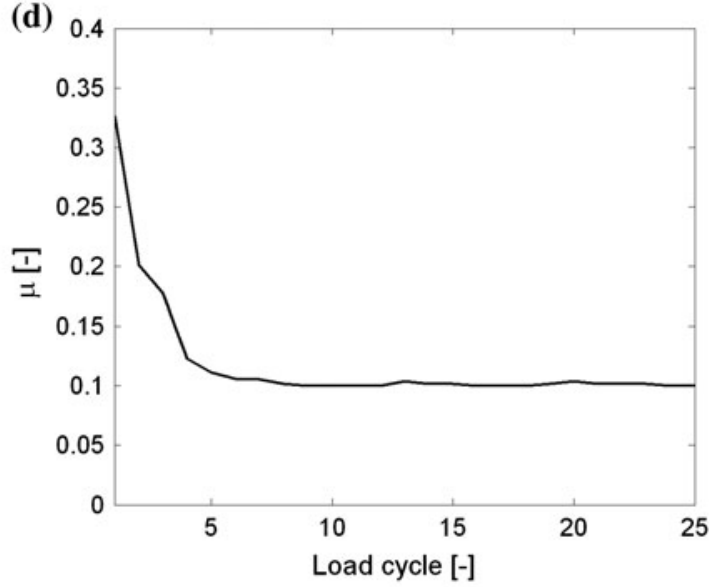

(f)

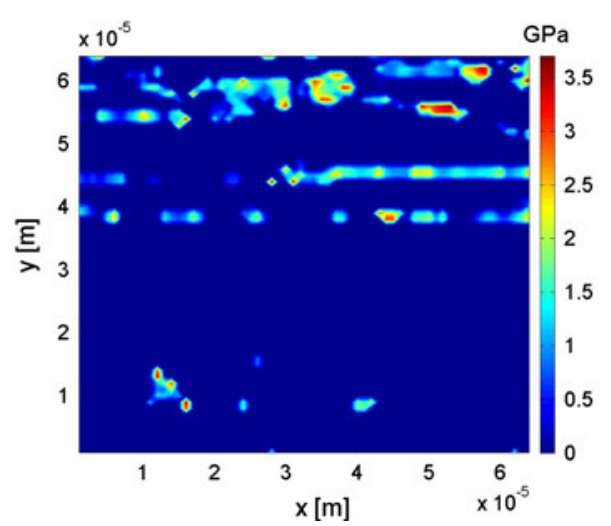

cycle. d Coefficient of friction versus load cycle. e Local coefficient of friction at cycle 25 (black no contact, white high friction, gray low friction. f Pressure field 
resistance, however, looking at the friction coefficient it can be seen the coefficient of friction remains at a relatively high level (0.17), due to the fact that the surface stays relatively rough and the oil cannot provide sufficient protection at all contact locations. If the friction level remains at this high value more energy will be dissipated and thus available for inducing wear which is not wanted in
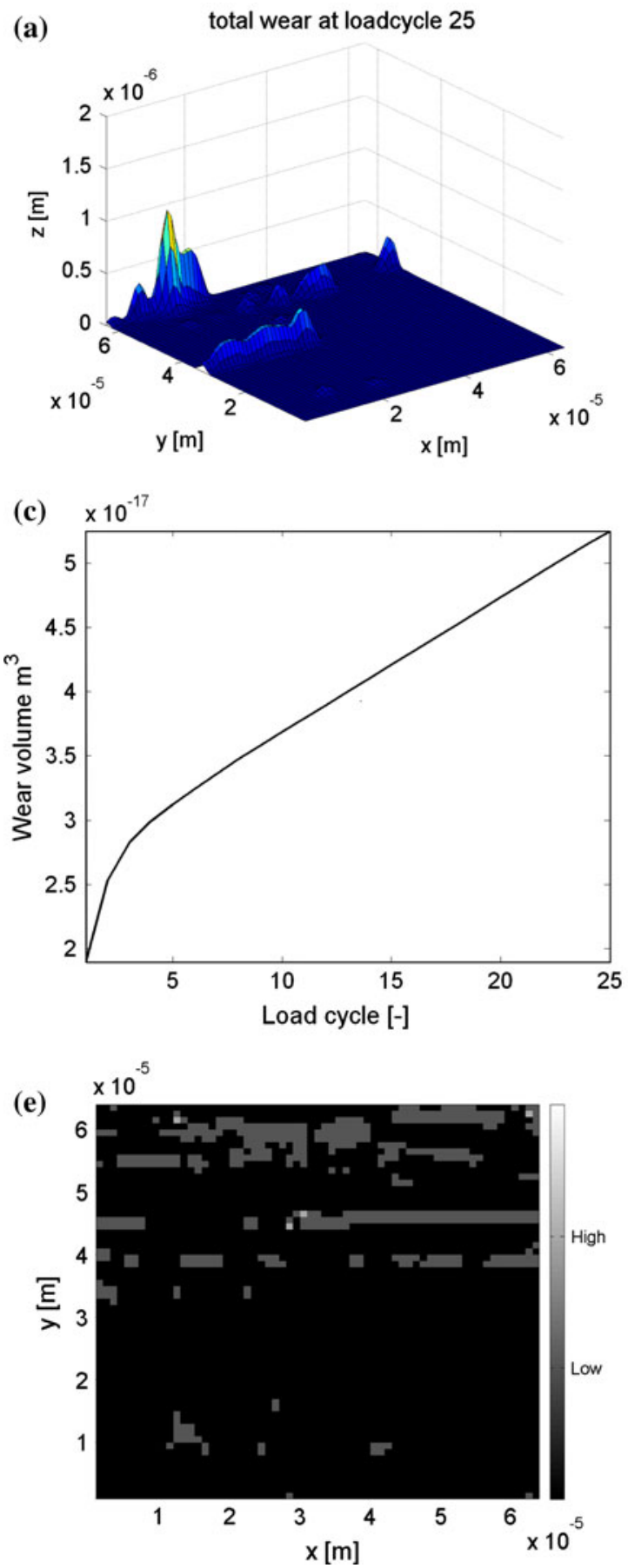

Fig. 16 Results for the simulation with material curve $B$ with $\varepsilon_{\mathrm{pmax}}=5 \%$. a Wear volume after 25 cycles. b Original surface geometry (transparent) versus worn surface (solid). c Wear volume as a system. The efficiency of the system itself will also be reduced by the higher frictional level. However, since mild wear is currently not modeled the exact influence of the higher friction level is difficult to quantify. However, higher friction levels induce higher contact temperatures and a more tribologically stressed system. As the removal rate of the chemical layer is higher it can be suggested that
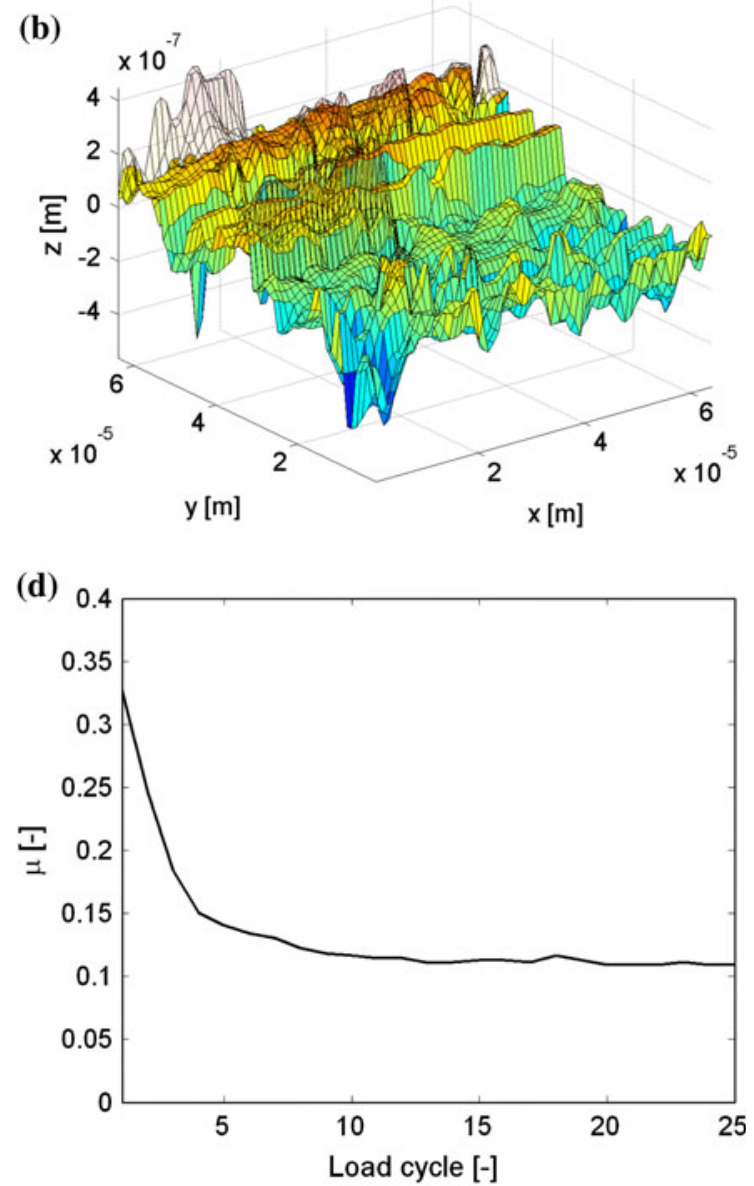

(f)

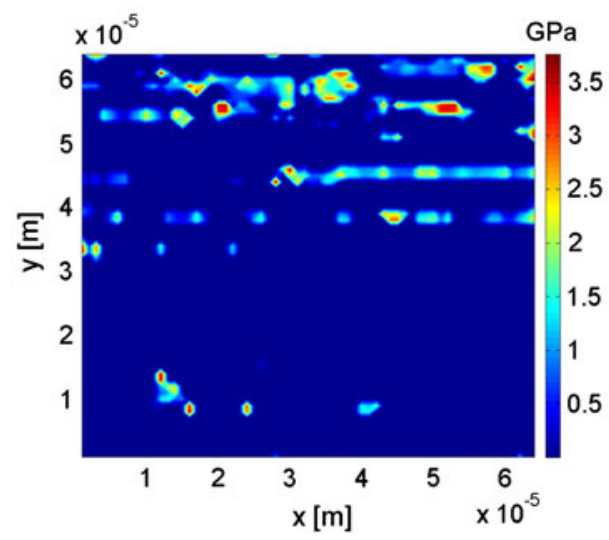

a function of load cycle. d Coefficient of friction versus load cycle. e Local coefficient of friction at cycle 25 (black no contact, white high friction, gray low friction. f Pressure field 
the wear level of this type of system will be increased by a more corrosive wear mechanism.

In the second calculation example the material model curve A is used see Fig. 9. The results of the simulation are given in Fig. 14 and it can be concluded that the wear volume here is increasing in a linear manner after it is

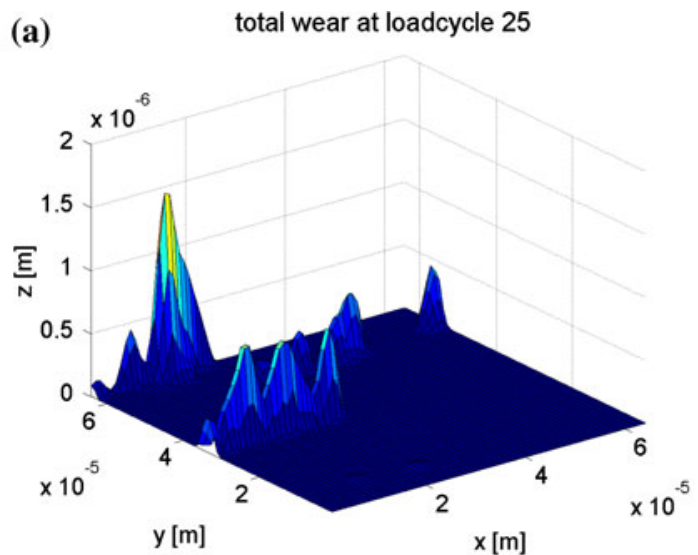

(c)
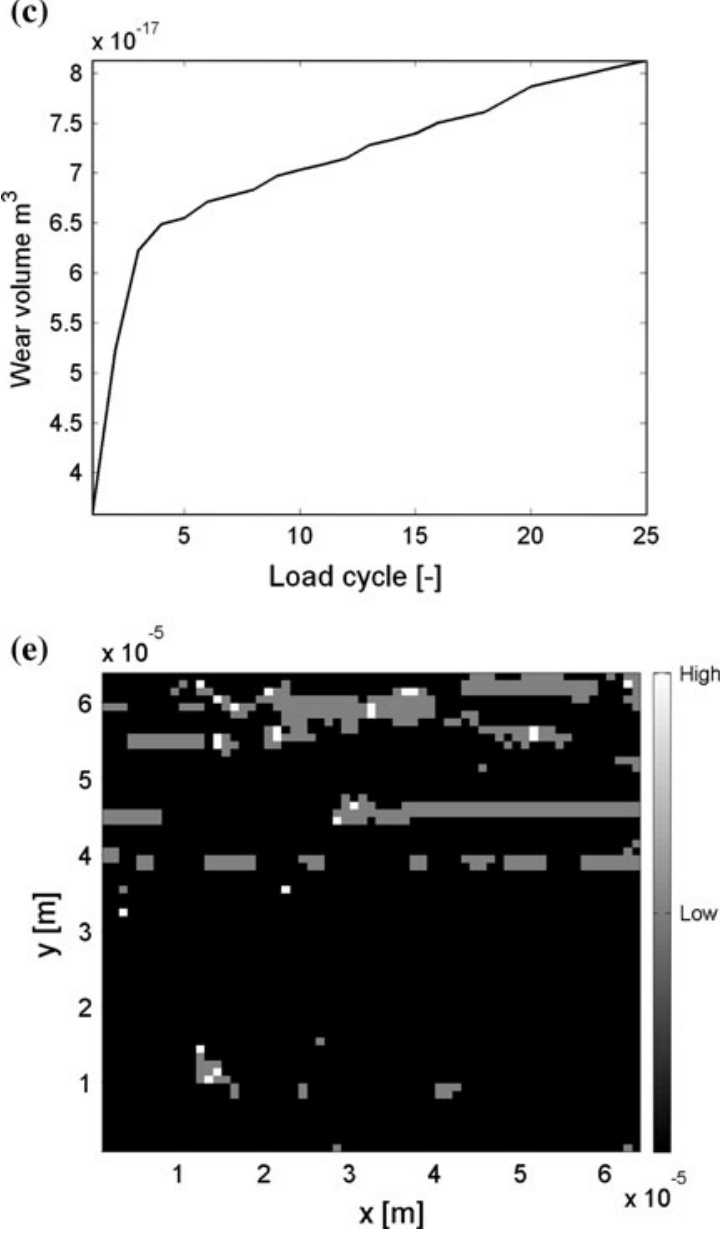

Fig. 17 Results for the simulation with only hardening toward the surface. a Wear volume after 25 cycles. b Original surface geometry (transparent) versus worn surface (solid). c Wear volume as a function stabilized after the first load cycles. This suggests that the surface is still smoothening (running-in) after 25 cycles. This is also represented in a lowering of the overall coefficient of friction. This suggests that the surface roughness is reduced more than when bulk material would be present at the surface instead of the NC-layer, which reduces the

(b)
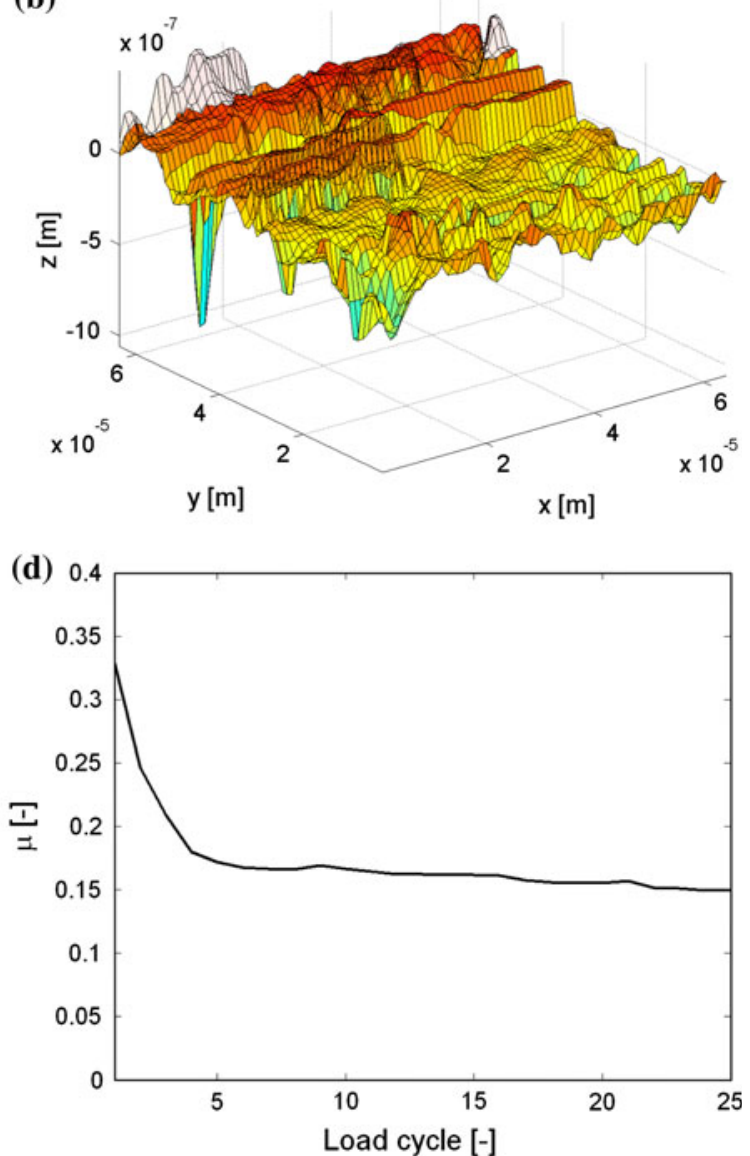

(f)

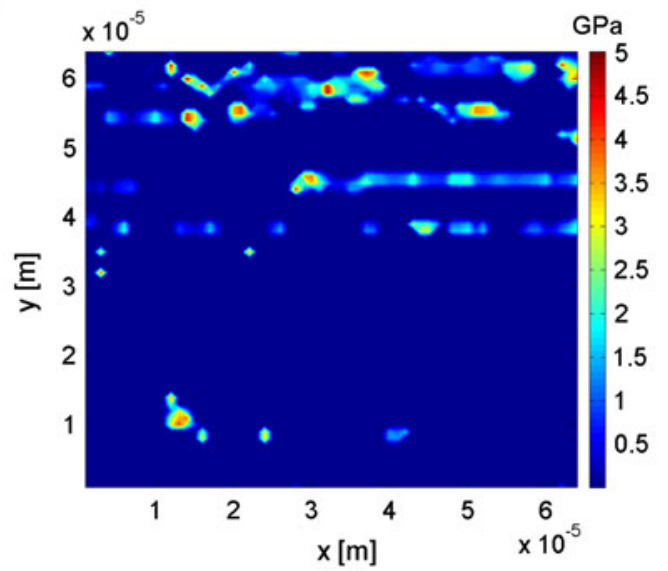

of load cycle. d Coefficient of friction versus load cycle. e Local coefficient of friction at cycle 25 (black no contact, white high friction, gray low friction. $\mathbf{f}$ Pressure field 
stress put on the chemical protective layer. The effect of a NC-layer on top of the bulk material would in this case be isolating the plastic core into the surface material ensuring that material removal will result in a smoothening of the surface and thus local pressure reduction, which will reduce wear on the larger time scale. At first sight the presence of a NC-layer with the properties of material curve A would not be beneficial; however, with respect to the lifetime of the component it will be. Especially if the ductility would be increased due to super plasticity, however this concept is not fully understood in NC materials yet and is thus left out of the discussion for this moment.

Calculation of the frictional and wear behaviors using curve $\mathrm{B}$ or $\mathrm{C}$ (which is very close to curve $\mathrm{B}$ ) as the material model for the NC-layer one finds that the effect of roughness reduction is even more pronounced, see Fig. 15, and the system and the overall fictional level is reduced even further for the complete contact, e.g., the oil is capable of protecting the complete system. To the authors opinion this is the system strived for by nature, since the least amount of energy is dissipated through frictional losses. However, as can be concluded from the figure the wear volume is still increasing linearly after 25 cycles while the complete surface is protected by the chemical layer which is most probably due to the very strictly chosen $\varepsilon_{\mathrm{pl}_{\mathrm{eq}} \max }=2 \%$ for the NC-material. In literature both high- and low-equivalent strain values are reported for the NC-layer. For this purpose a simulation is run where the maximum equivalent strain is increased to $\varepsilon_{\mathrm{pl}_{\mathrm{eq}} \mathrm{max}}=5 \%$, the results of which are presented in Fig. 16. The effect of increasing the strain is mainly limited to the first five cycles after which the slope of the wear volume increase is stabilized at the same value as for the simulations run with an equivalent strain of $\varepsilon_{\mathrm{pl}_{\mathrm{eq}} \max }=2 \%$.

If the hardness curve showing only hardening within the NC-layer is used, the results for the calculations are given in Fig. 17. The wear volume is in the same range as for the bulk material model, with the difference that the wear is still slowly increasing after 25 cycles, suggesting a slowly smoothening surface. This effect will lower the local pressure put on the protecting layer, decreasing the overall coefficient of friction. This mechanism would very well be the most beneficial for the system since the removal rate is low compared to the ones obtained using a soft ductile NC-layer and the while still effectively reducing the local pressure by roughness reduction, resulting in mild running conditions and as a result a low wearing system without a large volume loss of material.

\section{Conclusions}

A wear model is presented which is based on the hypothesis that a system which wears under mild oxidative conditions is protected by a surface layer of which the removal and grow rate are in balance. The values used as an input for this model are taken from literature and are therefore realistic values. To investigate the effect the different material properties of the NC-layer, reported throughout literature, has on the wear of this type of system three different material behaviors are included in the model. From the results it can be concluded that the presence of a NC-layer has a great influence on the wear behavior and frictional behavior of the system. The most beneficial would be a NC-layer with increased hardness toward the surface while also presenting a slight increase of ductility (maximum equivalent strain set at $2 \%$ ). The real properties of the NC-layer are still unclear yet and future research on this will hopefully give a better insight into the properties and origin of this important layer.

Open Access This article is distributed under the terms of the Creative Commons Attribution Noncommercial License which permits any noncommercial use, distribution, and reproduction in any medium, provided the original author(s) and source are credited.

\section{Appendix}

\section{Surface Displacement Coefficients}

The surface displacement due to a unit pressure put on a surface patch of size $2 a \times 2 b$ [38]:

$$
D_{33}^{n}=\frac{1}{\pi E}\left\{\begin{array}{c}
(x+a) \ln \left(\frac{(y+b)+\sqrt{(y+b)^{2}+(x+a)^{2}}}{(y-b)+\sqrt{(y-b)^{2}+(x+a)^{2}}}\right)+(y+b) \ln \left(\frac{(x+a)+\sqrt{(y+b)^{2}+(x+a)^{2}}}{\left((x-a)+\sqrt{(y+b)^{2}+(x-a)^{2}}\right.}\right)+ \\
(x-a) \ln \left(\frac{(y-b)+\sqrt{(y-b)^{2}+(x-a)^{2}}}{(y+b)+\sqrt{(y+b)^{2}+(x-a)^{2}}}\right)+(y-b) \ln \left(\frac{(x-a)+\sqrt{(y-b)^{2}+(x-a)^{2}}}{(x+a)+\sqrt{(y-b)^{2}+(x+a)^{2}}}\right)
\end{array}\right\}
$$


The surface displacement due to unit traction put on a surface patch of size $2 a \times 2 b$ [38]:

$D_{31}^{n}=\frac{1-2 v}{4 \pi G}\left\{\begin{array}{c}(y+b) \ln \left(\frac{\sqrt{(x+a)^{2}+(y+b)^{2}}}{\sqrt{(x-a)^{2}+(y+b)^{2}}}\right)+(y-b) \ln \left(\frac{\sqrt{(x-a)^{2}+(y-b)^{2}}}{\sqrt{(x+a)^{2}+(y-b)^{2}}}\right)+ \\ (x+a)\left(\arctan \frac{y+b}{x+a}-\arctan \frac{y-b}{x+a}\right)+(x-a)\left(\arctan \frac{y-b}{x-a}-\arctan \frac{y+b}{x-a}\right)\end{array}\right\}$

Expressions for the surface displacement due to a volume element of size $2 a \times 2 b \times 2 c$ with uniform strains inside the volume [27]:

$K_{i j}=-\left.\left.\left.\frac{1}{\pi}\left[\begin{array}{l}-2 \varepsilon_{13}\left(R_{, 133}-\phi_{, 1}\right)-2 \varepsilon_{23}\left(R_{, 233}-\phi_{, 2}\right)+2 \varepsilon_{12}\left(D \psi_{, 12}+R_{, 123}\right) \\ +\varepsilon_{11}\left(D \psi_{, 11}+R_{, 113}\right)+\varepsilon_{22}\left(D \psi_{, 22}+R_{, 223}\right) \\ +\varepsilon_{33}\left((2-3) \phi_{, 3}+R_{, 333}\right)-2 v \varepsilon_{i i} \phi_{3}\end{array}\right]\right|_{-a} ^{a}\right|_{-b} ^{b}\right|_{-c} ^{b}$

$$
\begin{aligned}
S_{113}^{\text {elas }}(x, y, z) & =2 v \tan ^{-1}\left(\frac{z^{2}+y^{2}-R y}{z x}\right) \\
+2(1 & -v) \tan ^{-1}\left(\frac{R-y+z}{x}\right)+\frac{x y z}{R\left(x^{2}+z^{2}\right)} \\
S_{223}^{\text {elas }}(x, y, z) & =2 v \tan ^{-1}\left(\frac{z^{2}+y^{2}-R y}{z x}\right) \\
+2(1 & -v) \tan ^{-1}\left(\frac{R-x+z}{y}\right)+\frac{x y z}{R\left(y^{2}+z^{2}\right)} \\
S_{333}^{\text {elas }}(x, y, z) & =\tan ^{-1}\left(\frac{z^{2}+y^{2}-R y}{z x}\right) \\
-\frac{x y z}{R} & \left(\frac{1}{x^{2}+z^{2}}+\frac{1}{y^{2}+z^{2}}\right) \\
S_{123}^{\text {elas }}(x, y, z) & =-\frac{z}{R}-(1-2 v) \ln (R+z) \\
S_{133}^{\text {elas }}(x, y, z) & =\frac{z^{2} y}{R\left(x^{2}+z^{2}\right)} \\
S_{233}^{\text {elas }}(x, y, z) & =\frac{z^{2} x}{R\left(y^{2}+z^{2}\right)}
\end{aligned}
$$

$$
S_{i j k l}^{\mathrm{pl}}=v f\left(\varepsilon_{i j}\right)_{k, k m m} \delta_{i j}-f\left(\varepsilon_{i j}\right)_{k, k i j}+\left.\left.\left.(1-v)\left(f\left(\varepsilon_{i j}\right)_{i, k k j}+f\left(\varepsilon_{i j}\right)_{j, k k i}\right)\right|_{-a} ^{a}\right|_{-b} ^{b}\right|_{-c} ^{c}
$$
volume:

The stress influence vector can be split up into two parts: one for the stress inside the plastic volume $\Omega_{\mathrm{p}}$ and one outside the volume. The stresses outside the plastic

$$
\begin{aligned}
S_{111}^{\text {elas }}(x, y, z) & =\frac{-z}{R}\left[1+\frac{y z-x^{2}}{(R+z)(R-y)}\right] \\
+2 v\left(\frac{y}{(R+z)}\right)-2 \ln (R-y) & \\
S_{221}^{\text {elas }}(x, y, z) & =\frac{-y z}{(R(R+z))}-2 v\left(\frac{y}{R+z}+\ln (R-y)\right) \\
S_{331}^{\text {elas }}(x, y, z) & =\frac{x y^{2} R}{R\left(x^{2}+z^{2}\right)} \\
S_{121}^{\text {elas }}(x, y, z) & =\frac{-z x}{(R(R+z))}-2 v\left(\frac{x}{(R+z)}\right)-\ln (R-x) \\
S_{131}^{\text {elas }}(x, y, z) & =\frac{x y z}{R\left(x^{2}+y^{2}\right)}+\tan ^{-1}\left(\frac{z^{2}+y^{2}-y R}{x z}\right) \\
S_{231}^{\text {elas }}(x, y, z) & =\frac{-z}{R}
\end{aligned}
$$


The stress inside the volume:

$S_{i j k l}^{\mathrm{pl}}=v f\left(\varepsilon_{i j}\right)_{k, k m m} \delta_{i j}-f\left(\varepsilon_{i j}\right)_{k, k i j}+(1-v)\left(f\left(\varepsilon_{i j}\right)_{i, k k j}+f\left(\varepsilon_{i j}\right)_{j, k k i}\right)-\mu 2 \varepsilon_{i j}-\left.\left.\left.\lambda \varepsilon_{k k} \delta_{i j}\right|_{-a} ^{a}\right|_{-b} ^{b}\right|_{-c} ^{c}$

Here is $\delta_{i j}$ the kronecker delta and $\mu=\frac{E}{2(1+v)}$ with the following definition of the derivatives of $f_{i j k l}$ :

$$
\begin{aligned}
& f_{i, j j} / C=2 \varepsilon_{1 i}\left(\phi_{, 1}^{I}+\phi_{, 1}\right)+2 \varepsilon_{2 i}\left(\phi_{, 2}^{I}+\phi_{, 2}\right) \\
& +2 \varepsilon_{3 i}\left(\phi_{, 3}^{I}-\phi_{, 3}\right) \\
& (i=1 \text { or } 2) \\
& f_{3, j j} / C=2 \varepsilon_{13}\left(\phi_{, 1}^{I}+4 x_{3}-4 R_{, 133}+3 \phi_{, 1}\right) \\
& +2 \varepsilon_{23}\left(\phi_{, 2}^{I}+4 x_{3} \phi_{, 23}-4 R_{, 233}+3 \phi_{, 2}\right) \\
& +8 \varepsilon_{12}\left(-x_{3} \phi_{, 12}+R_{, 123}+D \psi_{, 12}\right) \\
& +4 \varepsilon_{11}\left(-x_{3} \phi_{, 22}+R_{, 113}+D \psi_{, 11}\right) \\
& +4 \varepsilon_{22}\left(-x_{3} \phi_{, 22}+R_{, 223}+D \psi_{, 22}\right)+2 \varepsilon_{33} \\
& \times\left(\phi_{, 3}^{I}-2 x_{3} \phi_{, 33}+2 R_{, 333}-(5-4 v) \phi_{, 3}\right)-8 v \phi_{, 3} \\
& f_{m, j j m} / C=4 \varepsilon_{13}\left(\phi_{, 13}^{I}-2 R_{, 1333}+2 x_{3} \phi_{, 133}+3 \phi_{, 13}\right) \\
& +4 \varepsilon_{23}\left(\phi_{, 23}^{I}-2 R_{, 2333}+2 x_{3} \phi_{233}+\phi_{, 23}\right) \\
& +4 \varepsilon_{12}\left(\phi_{, 12}^{I}+2 R_{, 1233}-2 x_{3} \phi_{, 123}+(1-4 v) \phi_{, 12}\right) \\
& +2 \varepsilon_{11}\left(\phi_{, 11}^{I}+2 R_{, 1133}-2 x_{3} \phi_{, 113}+(1-4 v) \phi_{, 11}\right) \\
& +2 \varepsilon_{22}\left(\phi_{, 22}^{I}+2 R_{, 2233}-2 x_{3} \phi_{, 223}+(1-4 v) \phi_{, 22}\right) \\
& +2 \varepsilon_{33}\left(\phi_{, 33}^{I}+2 R_{, 3333}-2 x_{3} \phi_{, 333}-(7-4 v) \phi_{, 33}\right) \\
& -8 v \varepsilon_{i i} \phi_{, 33}
\end{aligned}
$$

$$
\begin{aligned}
f_{j, j} / C= & 2 \varepsilon_{13}\left(R_{, 13}^{I}-(3-4 v) R_{, 13}+4(1-v) x_{3} \phi_{, 1}\right. \\
& \left.-2 x_{3} R_{, 133}+2 x_{3}^{2} \phi_{, 13}\right) \\
+ & 2 \varepsilon_{23}\left(R_{, 23}^{I}-(3-4 v) R_{, 23}+4(1-v) x_{3} \phi_{, 2}\right. \\
& \left.-2 x_{3} R_{, 233}+2 x_{3}^{2} \phi_{, 23}\right) \\
+2 & \varepsilon_{12}\left(R_{, 12}^{I}-\left(1-2 D^{2}\right) R_{, 12}+4(1-v) D\left(x_{3}+x_{3}^{\prime}\right)\right. \\
& \left.\times \psi_{, 12}+2 x_{3} R_{, 123}-2 x_{3}^{2} \phi_{, 12}\right)
\end{aligned}
$$

$$
\begin{aligned}
& +\varepsilon_{11}\left(R_{, 11}^{I}+\left(1-2 D^{2}\right) R_{, 11}+4(1-v) D\left(x_{3}+x_{3}^{\prime}\right)\right. \\
& \left.\psi_{,, 11}+2 x_{3} R_{, 113}-2 x_{3}^{2} \phi_{, 11}\right) \\
& +\varepsilon_{22}\left(R_{, 22}^{I}+\left(1-2 D^{2}\right) R_{, 22}+4(1-v) D\left(x_{3}+x_{3}^{\prime}\right)\right. \\
& \left.\times \psi_{, 22}+2 x_{3} R_{, 2213}-2 x_{3}^{2} \phi_{, 22}\right) \\
& +\varepsilon_{33}\left(R_{, 33}^{I}+(3-4 v) R_{, 33}+8(1-v) x_{3} \phi_{, 3}\right. \\
& \left.-4(1-v) D \phi+2 x_{3} R_{, 333}-2 x_{3}^{2} \phi_{, 33}\right) \\
& -2 v \varepsilon_{i i}\left(\phi^{I}+(3-4 v) \phi+2 x_{3} \phi_{, 3}\right)
\end{aligned}
$$

Here the functions $R, \psi, \phi$ are defined as $R=$ $\sqrt{\left(x-x^{\prime}\right)+\left(y-y^{\prime}\right)+\left(z-z^{\prime}\right)}, \psi=\ln \left(R+\zeta_{3}\right)$, and $\phi$ $=1 / R$. The derivatives of this functions are given using $k \neq j \neq i$ and $\zeta_{1}=x-x^{\prime}, \zeta_{2}=y-y^{\prime}, \zeta_{3}=z-z^{\prime}$ (convolution/infinite space) or $\zeta_{3}=z+z^{\prime}$ (correlation/ halfspace) which is indicated by either a superscript $I$ or absence of one:

$\phi_{, k}=\zeta_{j} \ln \left[r+\zeta_{l}\right]+\zeta_{l} \ln \left[r+\zeta_{j}\right]-\zeta_{k} U_{k}$

$\phi_{, k l}=\ln \left[r+\zeta_{j}\right] \quad \phi_{, k k}=-U_{k} \quad \phi_{, k k k}=-\zeta_{i} V_{l}-\zeta_{l} V_{i}$

$\phi_{, k k l}=\zeta_{k} V_{k} \quad \phi_{, 123 k}=\frac{-\zeta_{k}}{r^{3}} \quad \phi_{, k k l l}=-\zeta_{k} \zeta_{l} W_{j}$

$\phi_{, k k k k}=\zeta_{l} \zeta_{k} W_{j}+\zeta_{k} \zeta_{j} W_{l} \quad \phi_{, k k k l}=V_{j}-\zeta_{k}^{2} W_{j}$

$R_{, k k l}=\zeta_{k} \ln \left[r+\zeta_{j}\right] \quad R_{, k k k}=\zeta_{i} \ln \left[r+\zeta_{l}\right]+\zeta_{l} \ln \left[r+\zeta_{j}\right]$

$$
-2 \zeta_{k} U_{k}
$$

$R_{, 123 k}=\frac{\zeta_{k}}{r} \quad R_{, k k k l}=\ln \left[r+\zeta_{j}\right]+\zeta_{k}^{2} V_{j} \quad R_{, k k l l}=\zeta_{k} \zeta_{l} V_{j}$

$R_{, k k k k}=-\zeta_{j} \zeta_{k} V_{l}-\zeta_{l} \zeta_{k} V_{j}-2 U_{k} \quad R_{, 123 k l}=\frac{-\zeta_{k} \zeta_{l}}{r^{3}}$

For $\psi$ and $\zeta_{3} \psi$ the derivative with respect to $\zeta_{3}$ become $\phi$ and $\left(\psi+R_{3}\right)$, respectively, so in the following only indices $k$ and $l$ are used which are both different and have the value of 1 or 2 : 


$$
\begin{aligned}
& \psi_{, k k}=-\zeta_{k} \ln \left[r+\zeta_{l}\right]-2 \zeta_{3} X_{k} \quad \psi_{, 12}=\zeta_{3} \ln \left[r+\zeta_{3}\right]-r \\
& \psi_{, k k k}=-2 \ln \left[r+\zeta_{l}\right]-\left(\zeta_{k}^{2}+\zeta_{3}^{2}\right) V_{l}-\left(\zeta_{3}-r\right) \zeta_{l} V_{3} \\
& \psi_{, k k l}=-\frac{\zeta_{k}}{r+\zeta_{3}} \\
& 4\left(\zeta_{3} \psi\right)_{, k k k}=2 \zeta_{l} \ln \left[r+\zeta_{3}\right]-\zeta_{3}\left(\zeta_{k}^{2}+\zeta_{3}^{2}\right) V_{l}-\zeta_{3} \zeta_{l}\left(\zeta_{3}-r\right) \\
& \times V_{3}-4 \zeta_{k} U_{k} \\
& 2\left(\zeta_{3} \psi\right)_{, k k l}=\zeta_{k} \ln \left[r+\zeta_{3}\right]-\frac{\zeta_{k} \zeta_{3}}{r+\zeta_{3}} \quad 2\left(\zeta_{3} \psi\right)_{, k k l l}=\zeta_{k} \zeta_{l} V_{3} \\
& +\frac{\zeta_{k} \zeta_{l} \zeta_{3}}{\left(r+\zeta_{3}\right)^{2} r} \\
& 4\left(\zeta_{3} \psi\right)_{4, k k l l}=V_{3} \zeta_{l} \zeta_{k}\left(\frac{\zeta_{3}}{r}-2\right)+\zeta_{3} \zeta_{k}\left[\zeta_{l}\left(\zeta_{3}-r\right) W_{3}-6 V_{l}\right. \\
& \left.+\left(\zeta_{k}^{2}+\zeta_{3}^{2}\right) W_{l}\right]-4 U_{k} \\
& 4\left(\zeta_{3} \psi\right)_{, k k k l}=2 \ln \left[r+\zeta_{3}\right]+\left(3 \zeta_{k}^{2}-2 \zeta_{3}^{2}\right) V_{3} \\
& +\zeta_{3} \zeta_{l}^{2}\left(\zeta_{3}-r\right) W_{3}+\frac{\zeta_{l}^{2} r+\left(\zeta_{k}^{2}+\zeta_{3}^{2}\right) \zeta_{3}}{r^{3}}
\end{aligned}
$$

The different functions used in (26) are:

$$
\begin{aligned}
U_{k} & =\tan ^{-1}\left[\frac{\zeta_{i} \zeta_{j}}{\zeta_{k} r}\right] \\
V_{k} & =\frac{1}{r\left(r+\zeta_{k}\right)} \\
W_{k} & =\frac{2 r+\zeta_{k}}{r^{3}\left(r+\zeta_{k}\right)^{2}} \\
X_{k} & =\tan ^{-1} \frac{\zeta_{k}}{\left(r+\zeta_{l}+\zeta_{j}\right)}
\end{aligned}
$$

\section{References}

1. Meng, H.C., Ludema, K.C.: Wear models and predictive equations their form and content. Wear 181-183, 443-457 (1995)

2. Archard, J.F.: Contact and rubbing of flat surfaces. J Appl Phys 24, 981-988 (1953)

3. Põdra, P., Andersson, S.: Simulating sliding wear with finite element method. Tribol Int 32, 71-81 (1999)

4. Olofsson, U., Andersson, S., Björklund, S.: Simulation of mild wear in boundary lubricated spherical roller thrust bearings. Wear 241, 180-185 (2000)

5. Sfantos, G.K., Aliabadi, M.H.: Wear simulation using an incremental sliding boundary element method. Wear 260, 1119-1128 (2006)

6. Shakhvorostov, D., Pohlmann, K., Scherge, M.: Structure and mechanical properties of tribologically induced nanolayers. Wear 260, 433-437 (2006)

7. Shakhvorostov, D., Gleising, B., Büscher, R., Dudzinski, W., Fischer, A., Scherge, M.: Microstructure of tribologically induced nanolayers produced at ultra-low wear rates. Wear 263, 1259-1265 (2007)

8. Hahn, H., Mondal, P., Padmanabhan, K.A.: Plastic deformation of nanocrystalline materials. Nanostruct Mater 9, 603-606 (1997)
9. Kato, H., Sasase, M., Suiya, N.: Friction-induced ultra-fine and nanocrystalline structures on metal surfaces in dry sliding. Tribol Int 43, 925-928 (2009)

10. Lu, L., Sui, M.L., Lu, K.: Superplastic extensibility of nanocrystalline copper at room temperature. Science 287, 1463-1466 (2000)

11. Schiotz, J., Tolla, F.D.D., Jacobsen, K.W.: Softening of nanocrystalline metals at very small grain sizes. Nature 391, 561-563 (1998)

12. Schiotz, J., Jacobsen, K.W.: A maximum in the strength of nanocrystalline copper. Science 301, 1357-1359 (2003)

13. Lu, L., Li, S.X., Lu, K.: An abnormal strain rate effect on tensile behavior in nanocrystalline copper. Scripta Mater 45, 1163-1169 (2001)

14. Wang, Z.B., Tao, N.R., Li, S., Wang, W., Lui, G., Lu, J., Lu, K.: Effect of surface nanocrystallization on friction and wear properties in low carbon steel. Mater Sci Eng 352, 114-119 (2002)

15. Wang, Y., Lei, T., Liu, J.: Tribo-metallographic behavior of high carbon steels in dry sliding: III. Dynamic microstructural changes and wear. Wear 231, 20-37 (1999)

16. Chen, X.H., Lu, J., Lu, L., Lu, K.: Tensile properties of a nanocrystalline 316 L austenitic stainless steel. Scripta Mater 52, 1039-1044 (2005)

17. Umemoto, M.: Nanocrystallization of steels by severe plastic deformation. Mater Trans 44, 1900-1911 (2003)

18. Garbar, I.: Microstructural changes in surface layers of metal during running-in friction processes. Meccanica 36, 631-639 (2001)

19. Nelias, D., Boucly, V., Brunet, M.: Elastic-plastic contact between rough surfaces: proposal for a wear or running-in model. J Tribol 128, 236-244 (2006)

20. Jacq, C., Nelias, D., Lormand, G., Girodin, D.: Development of a three-dimensional semi-analytical elastic-plastic contact code. J Tribol 124, 653-667 (2002)

21. Nelias, D., Antaluca, E., Boucly, V., Cretu, S.: A three-dimensional semianalytical model for elastic-plastic sliding contacts. J Tribol 129, 761-771 (2007)

22. Godfrey, D.: Boundary lubrication. Nasa Sp-118. 335-373 (1968)

23. Liu, S., Wang, Q.: Studying contact stress fields caused by surface tractions with a discrete convolution and fast Fourier transform algorithm. J Tribol 124, 36-45 (2002)

24. Kim, H.S., Bush, M.B.: The effects of grain size and porosity on the elastic modulus of nanocrystalline materials. Nanostruct Mater 11, 361-367 (1999)

25. Wang, F., Keer, L.M.: Numerical simulation for three dimensional elastic-plastic contact with hardening behavior. J Tribol 127, 494-502 (2005)

26. Bosman, R., Schipper, D.J.: Running in of metallic surfaces in the boundary lubricated regime. Wear (in review) (2010)

27. Liu, S.B., Wang, Q.: Elastic fields due to eigenstrains in a halfspace. J Appl Mech Trans ASME 72, 871-878 (2005)

28. Polonsky, I.A., Keer, L.M.: A numerical method for solving rough contact problems based on the multi-level multi-summation and conjugate gradient techniques. Wear 231, 206-219 (1999)

29. Zhang, Z., Yamaguchi, E.S., Kasrai, M., Bancroft, G.M.: Tribofilms generated from ZDDP and DDP on steel surfaces: part I. Tribol Lett 17, 211-220 (2005)

30. Ye, J.P., Araki, S., Kano, M., Yasuda, Y.: Nanometer-scale mechanical/structural properties of molybdenum dithiocarbamate and zinc dialkylsithiophosphate tribofilms and friction reduction mechanism. Jpn J Appl Phys 1 44, 5358-5361 (2005)

31. Martin, J.M., Grossiord, C., Le Mogne, T., Bec, S., Tonck, A.: The two-layer structure of zndtp tribofilms part 1: AES, XPS and XANES analyses. Tribol Int 34, 523-530 (2001)

32. Minfray, C., Martin, J.-M., Lubrecht, T., Belin, M., Mogne, T.L.: A novel experimental analysis of the rheology of ZDDP 
tribofilms, Tribology and Interface Engineering Series, pp. 807817. Elsevier, Amsterdam (2003)

33. So, H., Lin, Y.C.: The theory of antiwear for ZDDP at elevated temperature in boundary lubrication condition. Wear 177, 105-115 (1994)

34. Cutiongco, E.C., Chung, Y.-W.: Prediction of scuffing failure based on competitive kinetics of oxide formation and removal: application to lubricated sliding of AISI 52100 steel on steel. Tribology Transactions 37, 622-628 (1994)

35. Oila, A., Bull, S.J.: Assessment of the factors influencing micropitting in rolling/sliding contacts. Wear 258, 1510-1524 (2005)

36. Boucly, V.: Semi-analytical modeling of the transient-elasticplastic contact and its application to asperity collision, wear and running in of surfaces, PhD Thesis, Lyon, 2008 p 203

37. Evans, R.D.: Transmission Electron Microscopy of BoundaryLubricated Bearing Surfaces. Part: II Mineral Oil Lubricant with Sulfur and Phosphorus-Containing Gear Oil Additives. Tribology Trasnsactions 48, 299-307 (2005)

38. Willner, K.: Fully Coupled Frictional Contact Using Elastic Halfspace Theory. J Tribol 130, 031405-031408 (2008)

39. Nicholls, M.A., Norton, P.R., Bancroft, G.M., Kasrai, M., Do, T., Frazer, B.H., Stasio, G.D.: Nanometer scale chemomechanical characterization of antiwear films. Tribol Lett 17, 205-336 (2003)

40. Kasrai, M., Fuller, M.S., Bancroft, G.M., Yamaguchi, E.S., Ryason, P.R.: X-ray adsorption study of the effect of calcium sulfonate on antiwear film formation generated from neutral and basic ZDDPs: part 1-phosphorus species. Tribology Transactions 46, 434-442 (2003)

41. Nicholls, M.A., Do, T., Norton, P.R., Kasrai, M., Bancroft, G.M.: Review of the lubrication of metallic surfaces by zinc dialkyldithiophosphates. Tribol Int 38, 15-39 (2005)

42. Aktary, M., McDermott, M.T., McAlpine, G.A.: Morphology and nanomechanical properties of ZDDP antiwear films as a function of tribological contact time. Tribol Lett 12, 155-162 (2002)

43. Nicholls, M.A., Bancroft, G.M., Norton, P.R., Kasrai, M., De Stasio, G., Frazer, B.H., Wiese, L.M.: Chemomechanical properties of antiwear films using X-ray absorption microscopy and nanoindentation techniques. Tribol Lett 17, 245-259 (2004)

44. Ye, J.P., Kano, M., Yasuda, Y.: Evaluation of nanoscale friction depth distribution in ZDDP and MoDTC tribochemical reacted films using a nanoscratch method. Tribol Lett 16, 107-112 (2004)

45. Ji, H.B., Nicholls, M.A., Norton, P.R., Kasrai, M., Capehart, T.W., Perry, T.A., Cheng, Y.T.: Zinc-dialkyl-dithiophosphate antiwear films: dependence on contact pressure and sliding speed. Wear 258, 789-799 (2005)

46. Bec, S., Tonck, A., Georges, J.M., Coy, R.C., Bell, J.C., Roper, G.W.: Relationship between mechanical properties and structures of zinc dithiophosphate anti-wear films. In: Proceedings of the Royal Society of London Series A-Mathematical Physical and Engineering Sciences, vol 455, pp. 4181-4203, 1999

47. Komvopoulos, K., Do, V., Yamaguchi, E.S., Ryason, P.R.: Nanomechanical and nanotribological properties of an antiwear tribofilm produced from phosphorus-containing additives on boundary-lubricated steel surfaces. J Tribol Trans ASME 126, 775-780 (2004)

48. Warren, O.L.: Nanomechanical properties of films derived from zinc dialkyldithiophosphate. Tribol Lett 4, 189-198 (1998)

49. Minfray, C., Martin, J.M., Esnouf, C., Le Mogne, T., Kersting, R., Hagenhoff, B.: A multi-technique approach of tribofilm characterisation. Thin Solid Films 447, 272-277 (2004)

50. Pereira, G., Munoz-Paniagua, D., Lachenwitzer, A., Kasrai, M., Norton, P.R., Capehart, T.W., Perry, T.A., Cheng, Y.-T.: A variable temperature mechanical analysis of ZDDP-derived antiwear films formed on 52100 steel. Wear 262, 461-470 (2007)

51. Bancroft, G.M., Kasrai, M., Fuller, M., Yin, Z., Fyfe, K., Tan, K.H.: Mechanisms of tribochemical film formation: stabilityof tribo- and thermally-generated ZDDP films. Tribol Lett 3, 47-51 (1997)

52. Topolovec-Miklozic, K., Forbus, T., Spikes, H.: Film thickness and roughness of ZDDP antiwear films. Tribol Lett 26, 161-171 (2007) 